\title{
New Spectral Types L and T
}

\author{
J. Davy Kirkpatrick \\ Infrared Processing and Analysis Center, California Institute of Technology, \\ Pasadena, CA 91125; email: davy@ipac.caltech.edu
}

Key Words brown dwarfs, L dwarfs, low-mass stars, T dwarfs, Solar Neighborhood

Abstract The establishment of new spectral classes cooler than type $M$ has had a brief, yet already rich, history. Prototypes of the new "L dwarf" and "T dwarf" classes were first found in the late 1980s to mid-1990s, with a flood of new discoveries occurring in the late 1990s with the advent of deep, large-area, digital sky surveys. Over four hundred and fifty $\mathrm{L}$ and $\mathrm{T}$ dwarfs are now cataloged. This review concentrates on the spectroscopic properties of these objects, beginning with the establishment of classification schemes rooted in the MK Process. The resulting grid of spectral types is then used as a tool to ferret out the underlying physics. The temperature ranges covered by these spectral types, the complex chemical processes responsible for the shape of their emergent spectra, their nature as either true stars or brown dwarfs, and their number density in the Galaxy are discussed. Two promising avenues for future research are also explored: the extension of the classification system to three dimensions to account for gravity- and metallicity-dependent features, and the capability of newer large-area surveys to uncover brown dwarfs cooler than those now recognized.

\section{INTRODUCTION}

The need for new spectral types stems from the once frustrating but ultimately successful search for a theoretical entity termed a brown dwarf. Brown dwarfs, first postulated by Kumar (1963a,b) and Hayashi \& Nakano (1963), are low-mass by-products of star formation. During their pre-main-sequence contraction phases the cores of these low-mass objects become electron-degenerate, and it is this degeneracy that supports the object from collapsing further.

In objects of higher mass, contraction is halted not by electron degeneracy but by gas pressure; in those objects contraction produces core densities and temperatures capable of igniting hydrogen fusion, and this occurs before electron degeneracy is reached. These higher mass objects - known as dwarf stars - have been recognized since the dawn of Man because the Sun, as one prominent example, is rather hard to overlook. Over the past century and a half, astronomers have come to classify these dwarf stars based on their spectra, finally settling on a jumbled alphabet of $O B A F G K M$ to delineate the types, $O$ representing the hottest dwarfs and $M$ the coolest. 
The lack of stable hydrogen burning in brown dwarfs, on the other hand, makes them much dimmer intrinsically. Even twenty-five years after the theory was proposed, no definitive brown dwarfs had been found. Then, in 1988, a brown dwarf search finally revealed an object not classifiable as a normal M dwarf. In performing a near-infrared imaging search for low-mass companions around 200 white dwarfs, Becklin \& Zuckerman (1988) discovered a very red companion to the 32-pc distant DA4 white dwarf GD 165. A 6400-9000 A spectrum of the companion, dubbed GD 165B, was first obtained by Kirkpatrick, Henry, \& Liebert (1993). They noted that $\mathrm{TiO}$ absorption, which is the hallmark of the $\mathrm{M}$ spectral class, could not be distinguished even though VO may still have been present. Other observed absorption bands were found not to match the $\mathrm{CH}_{4} \mathrm{Or} \mathrm{NH}_{3}$ features seen in Jupiter and Saturn. Indeed the two independent temperature estimates for GD 165B (Zuckerman \& Becklin 1992; Kirkpatrick, Henry, \& Liebert 1993) supported a temperature lower than that of known $\mathrm{M}$ dwarfs but much hotter than the onset of $\mathrm{CO}$-to- $\mathrm{CH}_{4}$ conversion, prompting some researchers to believe that this object was a link between stars and planets. Other astronomers, however, seemed to write GD 165B off as an oddity.

A few years later, researchers uncovered another object whose status as a stellarto-planetary link was impossible to ignore. While searching an initial sample of 100 nearby stars with ages of $\sim 1$ Gyr, Nakajima et al. (1995) uncovered a common proper motion companion to the 5.7-pc distant M1 dwarf Gl 229. The implied distance of this companion, dubbed Gl 229B, meant that it was even dimmer than GD 165B. The most eye-opening revelation about $\mathrm{Gl} 229 \mathrm{~B}$ was that its near-infrared spectrum showed clear absorption by $\mathrm{CH}_{4}$ at $H$ and $K$ bands (Oppenheimer et al. 1995) and strongly resembled the spectrum of Jupiter. The temperature of $T_{\text {eff }}<$ $1000 \mathrm{~K}$ that was implied by the measured absolute luminosity and spectroscopic signatures bespoke a kind of cool object like none ever before seen outside our own Solar System.

In the years following these two initial discoveries, many similar objects were uncovered. Objects later in type than M9.5 dwarfs and spectroscopically akin to GD 165B were uncovered by a variety of techniques. The first of these was discovered by Kirkpatrick, Beichman, \& Skrutskie (1997) during a search of $105 \mathrm{deg}^{2}$ of data from the Two Micron All-Sky Survey (2MASS; M.F. Skrutskie, R.M. Cutri, R. Stiening, M.D. Weinberg, S. Schneider, et al., submitted) Prototype Camera. This was followed closely by the discovery of three more objects by Delfosse et al. (1997) in $230 \mathrm{deg}^{2}$ of data from the Deep Near-Infrared Survey (DENIS; Epchtein et al. 1999) and another by Ruiz, Leggett, \& Allard (1997) in a list of proper motion candidates pulled from $400 \mathrm{deg}^{2}$ of red optical data.

Soon thereafter, objects spectroscopically similar to Gl 229B were uncovered. The first two were discovered by Strauss et al. (1999) and Tsvetanov et al. (2000) in $400 \mathrm{deg}^{2}$ of Sloan Digital Sky Survey (SDSS; York et al. 1999) commissioning data, followed closely by four new objects discovered by Burgasser et al. (1999) in $1784 \mathrm{deg}^{2}$ of early 2 MASS data.

The beginnings of a spectral sequence were becoming clear, but a gap still remained between the GD 165B-type objects and those resembling Gl 229B. This 
gap was finally filled by Leggett et al. (2000) with the discovery of three objects from additional SDSS data. At last an entire spectral sequence had emerged linking the previously known low-mass stars to cool, planet-like (i.e., methane-bearing) objects.

\section{THE PHILOSOPHY OF CLASSIFICATION}

As these discoveries began to mount, it became obvious that new spectral types were needed. Spectra of objects like GD 165B were clearly distinct from previously known M dwarfs, and spectra of objects like Gl 229B were again clearly distinct from the others. Most researchers agreed that two new letters were needed for typing these objects; only Martín et al. (1997) advocated the use of a single new class. This suggests two immediate questions: (a) Which new letters should be chosen? (b) What sort of classification methodology would be best applied to these discoveries?

The first question is the easier one to answer. Suggestions for the two new letters were first put forth in the proceedings of a 1997 conference by Kirkpatrick (1998), who suggested " $\mathrm{H}$," "L," "T," and "Y" as the best contenders. The reasoning behind this was further chronicled in Kirkpatrick et al. (1999), who suggested specifically that "L" be used for GD 165B-like spectra and "T" for the Gl 229B-type. This decision was not reached in a vacuum; rather, before the choices were committed to print, many researchers in the brown dwarf community were polled about their preferences, members of IAU Commission 45 (who deal with issues related to stellar classification) were asked for their input, and audience response to the proposed letters was tested during various colloquia. As a result of this process, described in more detail in Kirkpatrick (2001a), there has been widespread, almost unanimous use of "L" and "T" as the spectral type descriptors for these objects.

A more divisive issue arises when trying to answer the second question, however. To this end, it is helpful to review the broader history of general scientific classification. The roots of modern classification are often traced back to the eighteenth century naturalist Carl Linnaeus, sometimes referred to as the Father of Taxonomy. His two-part naming of genus and species for categorizing plants is still used today to classify living organisms. Even though the concept of a binomial descriptor is still used, the basis for classification under the Linnaeus systemwhich considered only a very limited set of characteristics (the details of each plant's reproductive organs) - was found to produce "artificial" groupings. Instead, the methodology of classification most used today can be traced back to the seventeeth century naturalist John Ray. Ray's method drew upon all of the characteristics of a plant-leaves, roots, flowers, etc.-to determine classification. This methodology was accepted as producing the most believable "natural" groupings because it used the totality of information for each plant.

These historical facts also help reiterate why classification is important in the first place. Classification plays two critical roles. The first role is to help scientists uncover the underlying reasons why plants (or animals or stars, etc.) fall into the 
forms seen. In this sense classification is a means to an end; it is a necessary first step in understanding the reasons for the object's creation and/or makeup. Classification enables us to see the similarities and the differences more clearly. By having a clearer picture of the patterning of nature, the hope is that the underlying reasons for those similarities and differences will be easier to deduce. Linnaeus's sex-based system of typing was too focused on a single trait and sometimes placed objects that looked completely unalike into the same category. Ray's multi-trait morphological classification, on the other hand, proved more popular because it provided groupings seemingly more aligned with nature itself.

The second role of classification is to provide scientists with a vernacular. The Ray system lacked a quick shorthand for referring to each type of plant, a shortcoming that was remedied by Linnaeus. This quick shorthand makes scientific discussion much easier, whether the topic is lepus californicus or M5 dwarf stars. An essential element is that the vernacular be independent of our current, sometimes misguided, understanding of the biological or astrophysical underpinnings. Such a shorthand system of reference that is independent of theory and based only on observables will be immutable even as our ideas and perceptions of the underlying scientific causes change with time.

So, in answer to the second question, it would seem wise to follow these same principles in classifying $\mathrm{L}$ and $\mathrm{T}$ dwarfs. Fortunately, it is these principles that guide the MK system of classifcation (Morgan, Keenan, \& Kellman 1943), the most utilized system of spectral typing for normal stars. In explaining their use of standard reference points (i.e., actual stars) on the sky, Morgan \& Keenan (1973) state, in Ray-esque terms, that "these standard reference points do not depend on values of any specific line intensities or ratios of intensities; they have come to be defined by the appearance of the totality of lines, blends, and bands in the ordinary photographic region." They further state, "For example, a star located at A2 Ia would have a spectrum having a total appearance as in the standard $\alpha$ Cygni; a star of spectral type G2 V would have a spectrum whose appearance is similar to that of the Sun. The use of such a frame of reference makes the process of spectral classification a differential one." In an obituary to Morgan, Garrison (1995) explains further that "Morgan used the techniques of visual pattern recognition in a morphological approach to classification. In today's climate of the 'deification of quantification,' it is sometimes difficult for people to see the power of such an approach, yet the human brain has evolved to be ideally suited to such a methodology. Morgan was fond of using the analogy of the brain's ability to recognize familiar human faces. With pattern recognition, the result is immediate; nothing is measured, but all of the pattern information is compared with experience."

Not only does the MK system incorporate the totality of classification favored by the Ray approach, but it also parallels the Linnean ideal for naming. The MK system's convenient shorthand of spectral class plus luminosity class is ubiquitous in astronomy, from college introductory textbooks to premier research publications. 
Does the MK system also satisfy the two critical roles of classification, as outlined above? First, there is little doubt that the MK system is helpful to researchers studying the underlying reasons why stars appear as they do or else the system would not have had the longevity it has enjoyed. No Hertzprung-Russell diagram would be complete without a few MK classifications added. Second, its vernacular is widely used and its types have remained the same over the past sixty years. Mihalas (1984) points out that this constancy of types-a consequence of the fact that the classification grid is based on empirical standards-is the most important concept of the MK system "because it implies that the system does not, from the beginning, contain theoretical preconceptions. That's the absolute bane of trying to abstract anything from an empirical system. You build a theoretical preconception into it and all it does is give it back to you! This must be avoided at the outset."

In summary, the MK system satisfies all of the precepts of a robust and useful scientific taxonomy: ease of naming, classification based on the sum of the traits, utility for deducing the underlying physics, and shoptalk that is constant with time. In the following sections of this review, only those schemes rooted in the philosophy of the MK system will be discussed. Although others have proposed classifications in which the goal was to incorporate theory into the scheme (Basri et al. 2000) or in which a single index was the primary classification criterion (Martín et al. 1999), these schemes have been little used. Given the broader history of taxonomy, it is not surprising that MK-like schemes have been the ones most universally accepted.

\section{CORE CLASSIFICATION SCHEMES}

A total of $403 \mathrm{~L}$ dwarfs and $62 \mathrm{~T}$ dwarfs are currently known. Table 1 is provided as a historical reference to the discoveries found prior to the writing of this review. Spectroscopic features that distinguish these objects along with schemes used in their classification are discussed in sections that follow, beginning with the shortest observable wavelengths and moving longward.

\subsection{The Optical}

Optical spectra of a subset of bright $\mathrm{L}$ and $\mathrm{T}$ dwarfs are shown in Figure 1. Early-L dwarfs show a mélange of atomic and molecular bands, the most prominent being neutral alkali lines ( $\mathrm{Na} \mathrm{I,} \mathrm{K} \mathrm{I,} \mathrm{Rb} \mathrm{I,} \mathrm{Cs} \mathrm{I,} \mathrm{and} \mathrm{sometimes} \mathrm{Li} \mathrm{I),} \mathrm{oxide} \mathrm{bands} \mathrm{TiO}$ and $\mathrm{VO}$, hydride bands $\mathrm{CrH}$ and $\mathrm{FeH}$, and $\mathrm{CaOH}$. By mid-L the ground-state $\mathrm{Na} \mathrm{I}$ and $\mathrm{K} \mathrm{I}$ lines have grown tremendously in strength; the hydrides $\mathrm{MgH}, \mathrm{CaH}, \mathrm{CrH}$, and $\mathrm{FeH}$ have also strengthened, whereas the oxides $\mathrm{TiO}$ and $\mathrm{VO}$ have largely disappeared. By late- $\mathrm{L}$ and early- $\mathrm{T}, \mathrm{H}_{2} \mathrm{O}$ has increased in strength, the neutral alkali lines are still strong, and the hydrides are much reduced in prominence. By late- $\mathrm{T}, \mathrm{H}_{2} \mathrm{O}$ is a major absorber and the two prominent lines of $\mathrm{Na} \mathrm{I}$ and $\mathrm{K} \mathrm{I}$ have grown so wide that they have begun to further supress the pseudo-continuum between them at $\sim 7000 \AA$. 
TABLE 1 Discovery references for $\mathrm{L}$ and $\mathrm{T}$ dwarfs

\begin{tabular}{|c|c|c|c|}
\hline Reference & $\begin{array}{l}\text { \# of new } \\
L \text { dwarfs }\end{array}$ & $\begin{array}{l}\text { \# of new } \\
\text { T dwarfs }\end{array}$ & Note \\
\hline Becklin \& Zuckerman 1988 & 1 & 0 & GD $165 B$, the first L dwarf \\
\hline Nakajima et al. 1995 & 0 & 1 & Gl 229B, the first $T$ dwarf \\
\hline $\begin{array}{l}\text { Kirkpatrick, Beichman, \& } \\
\text { Skrutskie } 1997\end{array}$ & 1 & 0 & 2MASP J0345432 + 254023 \\
\hline Delfosse et al. 1997 & 3 & 0 & first DENIS L dwarfs \\
\hline $\begin{array}{l}\text { Ruiz, Leggett, \& Allard } \\
1997\end{array}$ & 1 & 0 & Kelu-1 \\
\hline Rebolo et al. 1998 & 1 & 0 & G 196-3B \\
\hline Martín et al. 1998 & 1 & 0 & Roque 25 \\
\hline Delfosse et al. 1999 & 1 & 0 & DENIS-P J0909 - 0658 \\
\hline Kirkpatrick et al. 1999 & 19 & 0 & more 2MASS L dwarfs \\
\hline Strauss et al. 1999 & 0 & 1 & SDSSp J162414.37 + 002915.6 \\
\hline Burgasser et al. 1999 & 0 & 4 & first 2MASS T dwarfs \\
\hline Cuby et al. 1999 & 0 & 1 & NTTDF 1205-0744 \\
\hline Zapatero Osorio et al. 1999 & 1 & 0 & SOri 47 \\
\hline Goldman et al. 1999 & 2 & 0 & $\begin{array}{l}\text { GJ 1001B, EROS-MP J0032 - } \\
4405\end{array}$ \\
\hline Martín et al. 1999 & 5 & 0 & more DENIS L dwarfs \\
\hline Reid et al. 2000 & 3 & 0 & more 2MASS L dwarfs \\
\hline Fan et al. 2000 & 7 & 0 & first SDSS L dwarfs \\
\hline Burgasser et al. 2000a & 0 & 1 & Gl 570D \\
\hline Tsvetanov et al. 2000 & 0 & 1 & SDSSp J134646.45 - 003150.4 ${ }^{\mathrm{c}}$ \\
\hline Leggett et al. 2000 & 0 & 3 & SDSS early-T "missing link" dwarfs \\
\hline Kirkpatrick et al. 2000 & 64 & 0 & more 2MASS L dwarfs \\
\hline Burgasser et al. 2000b & 0 & 1 & 2MASSI J0559191-140448 \\
\hline Gizis et al. 2000 & 4 & 0 & more 2MASS L dwarfs \\
\hline Zapatero Osorio et al. 2000 & 10 & 0 & more SOri L dwarfs \\
\hline $\begin{array}{l}\text { Gizis, Kirkpatrick, \& } \\
\text { Wilson } 2001\end{array}$ & 1 & 0 & GJ 1048B \\
\hline Wilson et al. 2001 & 3 & 0 & Gl 337C, G 618.1B, HD 89744B \\
\hline Burgasser et al. 2002a & 0 & 11 & more 2MASS T dwarfs \\
\hline Geballe et al. 2002 & 9 & $6^{\mathrm{a}}$ & more SDSS L and T dwarfs \\
\hline Schneider et al. 2002 & 8 & 0 & more SDSS L dwarfs \\
\hline Hall 2002 & 1 & 0 & 2MASSI J1315309 - 264951 \\
\hline
\end{tabular}


TABLE 1 (Continued)

\begin{tabular}{|c|c|c|c|}
\hline Reference & $\begin{array}{l}\text { \# of new } \\
L \text { dwarfs }\end{array}$ & $\begin{array}{l}\text { \# of new } \\
\text { T dwarfs }\end{array}$ & Note \\
\hline Potter et al. 2002 & 2 & 0 & Gl 564B and Gl 564C \\
\hline Liu et al. 2002a & 0 & 1 & IFA $0230-Z 1$ \\
\hline Liu et al. 2002b & 1 & 0 & Gl 779B \\
\hline Hawley et al. 2002 & 48 & 0 & more SDSS L dwarfs \\
\hline $\begin{array}{l}\text { Lodieu, Scholz \& } \\
\text { McCaughrean } 2002\end{array}$ & 3 & 0 & SSSPM L dwarfs \\
\hline Gizis 2002 & 9 & 0 & more 2MASS L dwarfs \\
\hline Dahn et al. 2002 & 1 & 0 & 2MASSW J2244316 + 204343 \\
\hline $\begin{array}{l}\text { Barrado y Navascués et al. } \\
2002\end{array}$ & 1 & 0 & SOri 71 \\
\hline Zapatero Osorio et al. 2002 & 0 & 1 & SOri 70 \\
\hline Scholz \& Meusinger 2002 & 1 & 0 & SSSPM J0829 - 1309 \\
\hline Liebert et al. 2003 & 1 & 0 & 2MASSI J0144353 - 071614 \\
\hline Burgasser et al. 2003c & 0 & 1 & 2MASS J15031961 + 2525196 \\
\hline Scholz et al. 2003 & 0 & 1 & $\epsilon$ Ind B \\
\hline Salim et al. 2003 & 1 & 0 & LSR $0602+3910$ \\
\hline Gizis et al. 2003 & 3 & 0 & more 2MASS L dwarfs \\
\hline Kendall et al. 2003 & 7 & 0 & more 2MASS L dwarfs \\
\hline Wilson et al. 2003 & 10 & 0 & more 2MASS L dwarfs \\
\hline Berriman et al. 2003 & 1 & 0 & 2MASSI J0104075 - 005328 \\
\hline Lépine, Rich \& Shara 2003 & 1 & 0 & LSR 1610 - 0040 (sdL?) \\
\hline Burgasser et al. 2003a & 1 & 0 & $\begin{array}{l}\text { 2MASS J05325346 + } 8246465 \\
\text { (sdL) }\end{array}$ \\
\hline $\begin{array}{l}\text { Thorstensen \& Kirkpatrick } \\
2003\end{array}$ & 1 & 0 & 2MASS J07003664 + 3157266 \\
\hline $\begin{array}{l}\text { Burgasser, McElwain \& } \\
\text { Kirkpatrick 2003d }\end{array}$ & 0 & 3 & more 2MASS T dwarfs \\
\hline Cruz et al. 2003 & 35 & 0 & more 2MASS L dwarfs \\
\hline Cruz et al. 2004 & 1 & 0 & 2MASS J05185995 - 2828372b \\
\hline Kendall et al. 2004 & 6 & 0 & more DENIS and 2MASS L dwarfs \\
\hline Burgasser et al. 2004a & 1 & 7 & more 2 MASS T dwarfs $+1 \mathrm{sdL}$ \\
\hline Knapp et al. 2004 & 22 & $12^{\mathrm{d}}$ & more SDSS L and T dwarfs \\
\hline Stanway et al. 2004 & 1 & 0 & GOODS-N $i$-drop \#2 \\
\hline Metchev \& Hillenbrand & 1 & 0 & HD 49197B \\
\hline
\end{tabular}


TABLE 1 (Continued)

\begin{tabular}{lccl}
\hline Reference & $\begin{array}{c}\text { \# of new } \\
\text { L dwarfs }\end{array}$ & $\begin{array}{l}\text { \# of new } \\
\text { T dwarfs }\end{array}$ & Note \\
\hline Burgasser 2004a & 1 & 0 & $\begin{array}{c}\text { 2MASS J16262034 }+3925190 \\
\text { (sdL) }\end{array}$ \\
Chauvin et al. 2004 & 1 & 0 & 2MASSW J1207334 - 393254B \\
Tinney et al. (submitted) & 0 & 5 & more 2MASS T dwarfs \\
Kirkpatrick et al. (in prep.) & 13 & 0 & more 2MASS L dwarfs \\
Cruz et al. (in prep.) & 22 & 1 & more 2MASS L and T dwarfs \\
Reid et al. (in prep.) & 61 & 0 & more 2MASS L dwarfs \\
Totals & $\mathbf{4 0 3}$ & $\mathbf{6 2}$ & \\
\hline
\end{tabular}

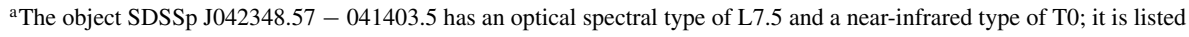
here as a $\mathrm{T}$ dwarf since this is its designation in the earliest reference.

${ }^{\mathrm{b}}$ This object has an optical type of L6.5 pec and appears to have an $\mathrm{L}+\mathrm{T}$ hybrid spectrum in the near-infrared; it has been listed here as an L dwarf.

${ }^{\mathrm{c}}$ This object was first published in Burgasser et al. (1999) but credit is given there to the SDSS team, who had discovered it earlier.

dOne of the "new" T dwarfs (SDSS J120747.17 + 024424.8, type T0) listed in the near-infrared spectroscopy paper by Knapp et al. (2004) appears in the optical spectroscopy paper by Hawley et al. (2002), where it is listed as an L8 dwarf. For bookkeeping purposes here, it is counted as an L dwarf via the earlier reference.

Note: Papers are ordered chronologically by publication date, even within a given year. Objects known to be close $\mathrm{L}+\mathrm{L}$, $\mathrm{L}+\mathrm{T}$, or $\mathrm{T}+\mathrm{T}$ binaries are listed in the table as one object, not two, except in the case of Gl 564BC for which separate spectra of both components have been acquired.

Note: Other objects in star formation regions such as Orion (Lucas et al. 2001) and Serpens (Lodieu et al. 2002) have been tentatively designated as L dwarfs via near-IR spectroscopy. These spectra are distinctly unlike normal L dwarfs and in some cases these same objects exhibit optical spectra consistent with M type. Late-M and L dwarfs of young age (low gravity) are discussed further in Section 5.2.

Note: Several possible L and T dwarf companions-to Gl 86 (Els et al. 2001), to LHS 2397a (Freed et al. 2003), and to 2MASSW J1047127 + 402644, 2MASSW J1426316 + 155701, 2MASSW J2140293 + 162518, and 2MASSW J2331016 - 1040618 (Close et al. 2003) —are not listed in the table because confirmation spectra have not yet been acquired. Furthermore, a possible T dwarf companion to the A dwarf double HD 150451AB (Carson et al. 2002) is not listed because it is now believed not to be a $\mathrm{T}$ dwarf even though common proper motion has been verified (Carson et al. in prep.).

Note: Bouy et al. (2003) estimate L spectral types for 31 objects using $I-J$ colors. A literature search shows that 18 of these have types measured from actual spectra, and a comparison to the Bouy et al. values shows that their estimated types are systematically too late by four subclasses on average. In individual cases these can be too late by as much as seven subclasses. Hence, most of the "new" L dwarfs listed in that paper are actually late M dwarfs. None of those L dwarfs are included here unless verified by actual spectra from other sources.

Note: A current list of $\mathrm{L}$ and $\mathrm{T}$ dwarfs can be found at http://DwarfArchives.org.

Figure 2 shows a finer grid of optical spectra from late-M through late-T, highlighting the region between 6800 and $8700 \AA$ in which a number of diagnostic features is found. The strong oxide bands seen in late-M dwarfs weaken through the early-L dwarfs. By mid-L strong absorption by neutral alkali lines and hydrides appears. By late- $\mathrm{L}$ types the ground-state $\mathrm{Na}$ I line is the major shaper of the emergent flux, and its influence continues through late-T.

Optical classifications in the spirit of the MK System have been established for L dwarfs by Kirkpatrick et al. (1999) and for T dwarfs by Burgasser et al. (2003b). 
TABLE 2 Anchor points for $\mathrm{L}$ and $\mathrm{T}$ dwarf classification

\begin{tabular}{|c|c|c|}
\hline $\begin{array}{l}\text { Spec. } \\
\text { type }\end{array}$ & Optical anchor & Near-IR anchor \\
\hline L0 & 2MASP J0345432 + 254023 & $\ldots$ \\
\hline L1 & 2MASSW J1439284 + 192915 & $\ldots$ \\
\hline L2 & Kelu-1 (at J13054019 - 2541059) & $\cdots$ \\
\hline L3 & 2MASSW J1146345 + 223053 & $\ldots$ \\
\hline L4 & 2MASSW J1155009 + 230706 & $\ldots$ \\
\hline L5 & DENIS-P J1228.2 - 1547 & $\ldots$ \\
\hline L6 & 2MASSs J0850359 + 105716 & $\ldots$ \\
\hline L7 & DENIS-P J0205.4 - 1159 & $\ldots$ \\
\hline L8 & 2MASSW J1632291 + 190441 & $\ldots$ \\
\hline T0 & - & SDSS J120747.17 + 024424.8 \\
\hline $\mathrm{T} 1$ & $\ldots$ & SDSSp J015141.69 + 124429.6 \\
\hline $\mathrm{T} 2$ & SDSSp J125453.90 - 012247.4 & SDSSp J125453.90 - 012247.4 \\
\hline $\mathrm{T} 3$ & - & 2MASS J12095613 - 1004008 \\
\hline $\mathrm{T} 4$ & $\ldots$ & 2MASSI J2254188 + 312349 \\
\hline T5 & 2MASSI J0559191 - 140448 & 2MASS J15031961 + 2525196 \\
\hline T6 & SDSSp J162414.37 + 002915.6 & SDSSp J162414.37 + 002915.6 \\
\hline $\mathrm{T} 7$ & - & 2MASSI J0727182 + 171001 \\
\hline $\mathrm{T} 8$ & 2MASSI J0415195 - 093506 & 2MASSI J0415195-093506 \\
\hline
\end{tabular}

Note: Anchors for the optical classification of L dwarfs are from Kirkpatrick et al. (1999); L dwarf anchors for the near-infrared have not yet been devised. T dwarf anchors are from Burgasser et al. (2003b) for the optical and Burgasser (priv. comm., collaborative results from the SDSS and 2MASS teams) for the near-infrared.

Both papers identified a set of reference objects (also known as anchor points) to serve as the on-sky classification standards for the typing scheme. These anchors are listed for convenience in Table 2. Although readers are referred to the above two papers for discussion of line/band strengths and spectral ratios useful for typing, it should be noted that the establishment of anchors is the single most important step in constructing a classification scheme. Details of how those anchors are used to classify a new set of objects-whether it be through automated methods or simply through by-eye fits (see, e.g., the discussion in Hawley et al. 2002) — are of secondary importance as long as those classifications are judged against the same set of standards.

The spectra of the L and T dwarfs shown in Figure 2 are those of the anchor points on the optical typing system. Except for the larger spectrum-to-spectrum difference between L8 and T2, these spectra can be viewed almost like consecutive frames in a movie. That is, if one were to stack plots of the individual spectra, one per page, and flip rapidly through the pages, the movie would flow from one frame 


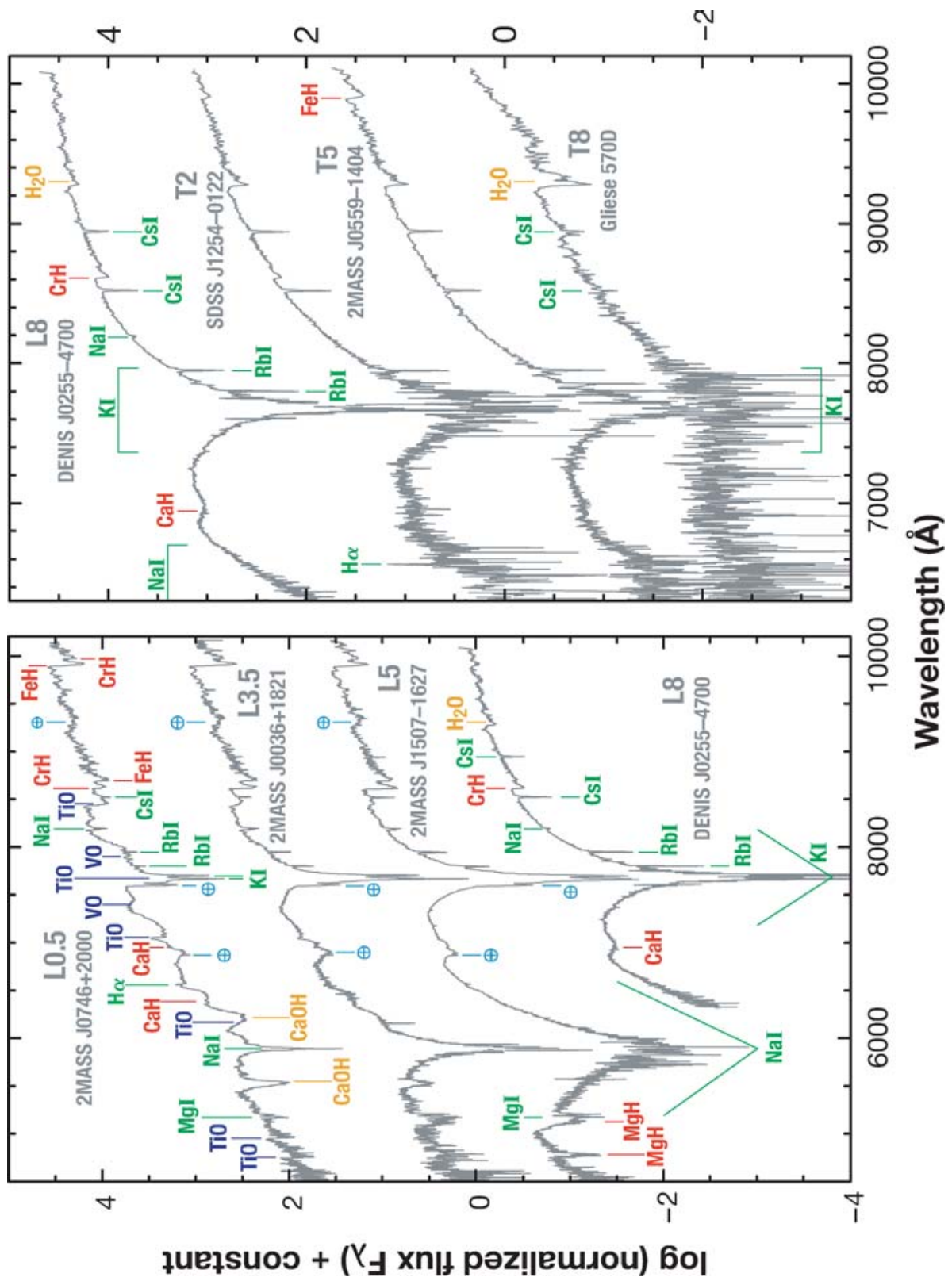




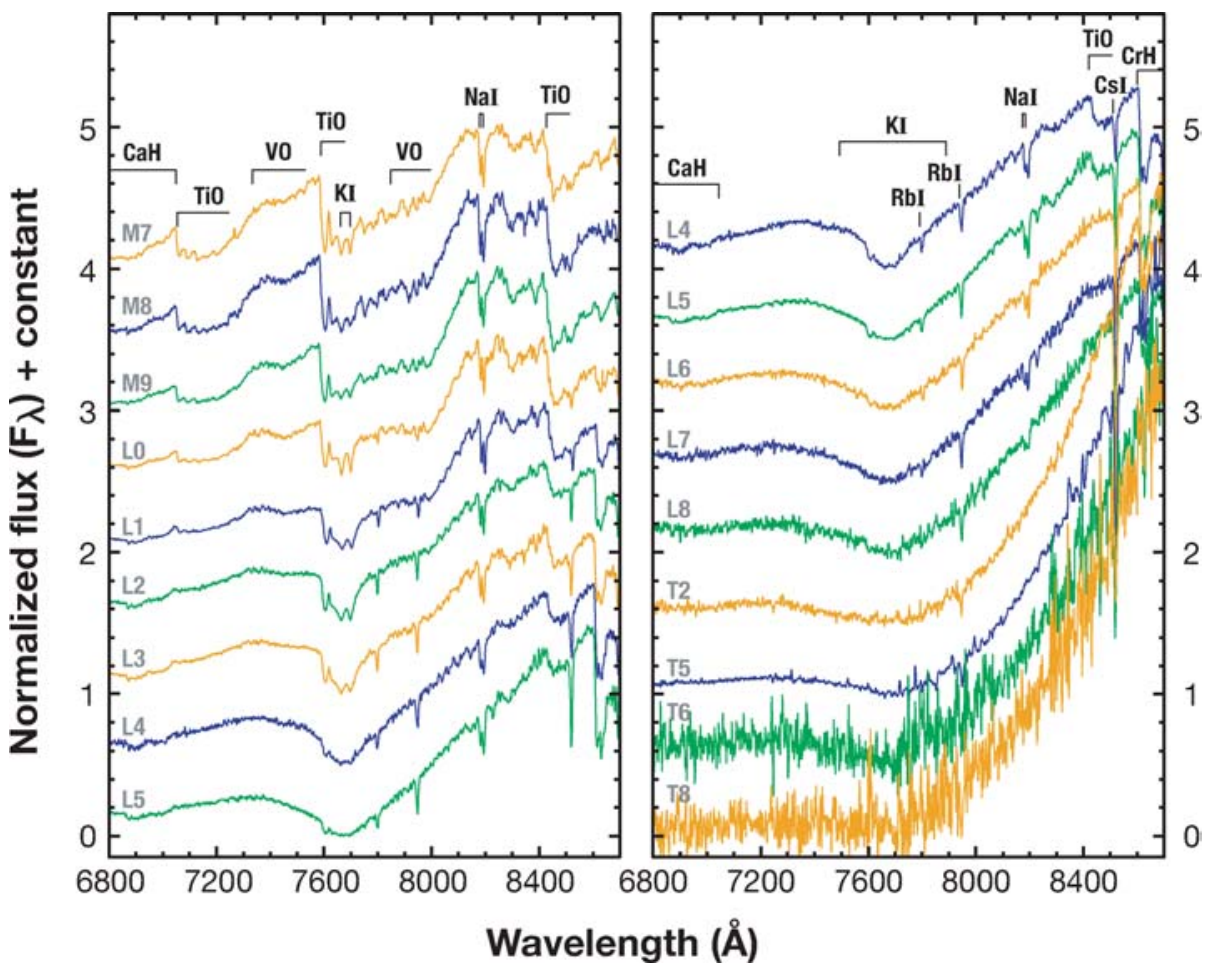

Figure 2 Detailed optical spectra from 6800 to $8700 \AA$ for a sequence of late-M through late-T dwarfs. The $\mathrm{L}$ and $\mathrm{T}$ spectra shown here are those of the $\mathrm{L}$ and $\mathrm{T}$ anchors (standards) from the optical classification system of Kirkpatrick et al. (1999) and Burgasser et al. (2003b). Spectra have been displaced vertically in half integral units to ease intercomparison.

to the next without any jumps in the sequence. Ideally, any grid of standards should show this same effect.

Currently, no optical spectra have been acquired that fill the aforementioned gap between L8 and T2. Oddly, the near-infrared spectral sequence (as discussed below) shows a continuum of spectra throughout this L8-T2 range without any

Figure 1 High signal-to-noise spectra of a sequence of bright L and T dwarfs. Feature identifications are given for alkali and other atomic lines in green, oxide bands in blue, hydride bands in red, and bands of $\mathrm{CaOH}$ and $\mathrm{H}_{2} \mathrm{O}$ in orange. For the L0.5, L3.5, and L5 spectra the contaminant telluric absorption bands are labelled in cyan; all other spectra have been corrected for telluric absorption. Left panel: 4500 to 10,100 ̊ spectra of a sequence of L dwarfs. Right panel: 6300 to 10,100 A spectra of a sequence of late-L through late-T dwarfs. These spectra were obtained at the W.M. Keck Observatory and are compiled from Reid et al. (2000), Burgasser et al. (2003b), and Kirkpatrick et al. (unpub.) and are displaced vertically by units of 1.5 in the log to ease intercomparison. 
gap. Note also in Figure 2 that there are fewer T-subtypes in the optical—only T2, T5, T6, and T8 - than there are in the near-infrared because the optical spectrumto-spectrum differences do not warrant a grid any finer than this. The T2, T5, T6, and T8 names for the optical subtypes were chosen to parallel the types of those same objects under the near-infrared scheme.

Large compilations of optical spectra of $\mathrm{L}$ and $\mathrm{T}$ dwarfs can be found in Kirkpatrick et al. (1999, 2000), Hawley et al. (2002), Burgasser et al. (2003b), Cruz et al. (2003), and the M, L, and T Dwarf Archives (http://DwarfArchives.org).

\subsection{The Near-Infrared}

Near-infrared (1.0-2.3 $\mu \mathrm{m})$ spectra of a subset of late-M through late-T spectra are shown in Figure 3 with a detail of the $0.95-1.35 \mu \mathrm{m}$ region shown in Figure 4. As seen in these plots, late- $\mathrm{M}$ and early- $\mathrm{L}$ spectra are characterized by strong bands of $\mathrm{H}_{2} \mathrm{O}$, bands of $\mathrm{FeH}$ and $\mathrm{CO}$, and neutral atomic lines of $\mathrm{Na}, \mathrm{Fe}, \mathrm{K}, \mathrm{Al}$, and $\mathrm{Ca}$. The hallmark of the $\mathrm{T}$ spectral class, $\mathrm{CH}_{4}$, appears at early- $\mathrm{T}$ along with strengthening $\mathrm{H}_{2} \mathrm{O}$. By late-T the near-infrared spectrum has been chewed up into a series of flux peaks near 1.08, 1.27, 1.69, and $2.08 \mu \mathrm{m}$, each of which has been sculpted on both sides by absorptions of $\mathrm{CH}_{4}$ and $\mathrm{H}_{2} \mathrm{O}$ (or, in the case of the short wavelength side of the $1.08-\mu$ m peak, by the broad wings of the K I line at $7800 \AA$ ). By $\mathrm{T} 8$ the $2.08-\mu \mathrm{m}$ peak has been further flattened by overlying collision-induced absorption by $\mathrm{H}_{2}$.

To date there has, oddly enough, still not been an independent spectral classification scheme established in the near-infrared for $\mathrm{L}$ dwarfs. ${ }^{1}$ All current systems either map near-infrared spectral ratios directly into the optical scheme or define classifications based only on spectral ratios of idealized spectral type standards and not on anchor objects. Geballe et al. (2002) show that their near-infrared scheme for T dwarfs (below) is extendable to L types, but the scheme is not optimized for L dwarfs and it lacks on-sky standards. Nevertheless, we utilize types from this scheme in subsequent sections because it is the one most widely used.

For $\mathrm{T}$ dwarfs, the two classification schemes in use are those of Burgasser et al. (2002a) and Geballe et al. (2002). The first of these employs anchor points on the sky, and the second employs a set of idealized ratios. Fortunately, both schemes yield very similar results and both groups are now combining their efforts to produce a single scheme that follows the MK Process (Burgasser, priv. comm.). The $\mathrm{T}$ dwarf anchor points for this collaborative scheme are listed in Table 2.

It should not be assumed a priori that a set of objects classified on optical spectroscopic morphology alone will necessarily fall into the same ordering based on near-infrared morphology. This is because the two spectral regions sample different physical environments in the atmosphere of the object. Nevertheless, many studies (e.g., Reid et al. 2001, Testi et al. 2001, McLean et al. 2003)

\footnotetext{
${ }^{1}$ McGovern et al. (priv. comm.) are currently working to remove this deficiency.
} 


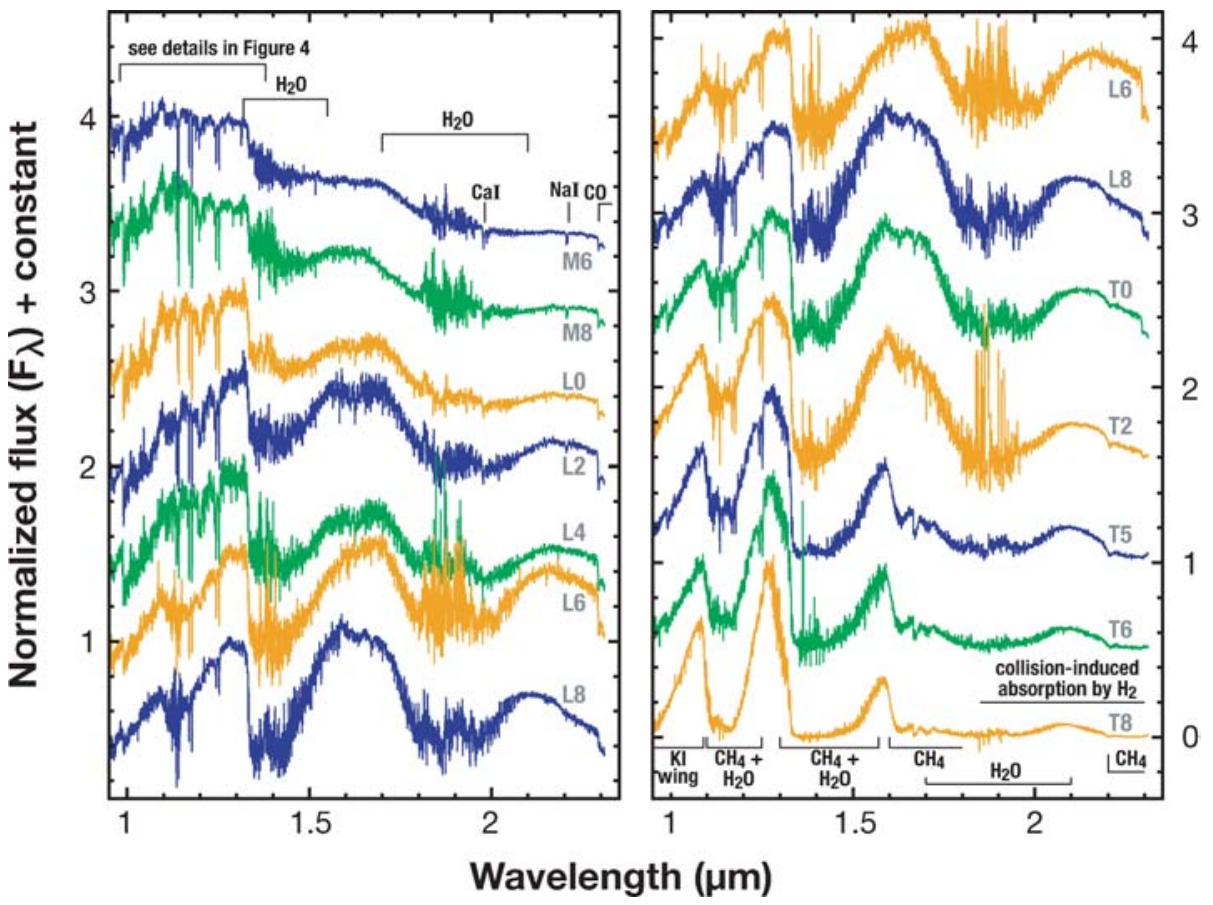

Figure 3 A sequence of mid-M through late-T spectra between 0.95 and $2.3 \mu \mathrm{m}$. Feature identifications within the $\mathrm{H}$ - and $\mathrm{K}$-band windows are marked. The feature-rich J-band spectra are shown on an expanded scale in Figure 4. Spectra have been displaced vertically by half integral units to ease intercomparison. These spectra come from the Brown Dwarf Spectroscopic Survey archive (http://www.astro.ucla.edu/ mclean/BDSSarchive/) and are from McLean et al. (2003).

have shown that optical L dwarf types usually map well into a near-infrared sequence.

There are, however, exceptions. Figure 3, which is a replotting of the spectral sequence shown in figure 3 of McLean et al. (2003), illustrates this point. The spectral types here are based on the optical classifications of these objects, and this leads to an ordering that lacks the "movie frame" consistency of the optical spectral sequence in Figure 2. If we consider only the sequence of J-band spectra in Figure 4, however, we see a much smoother ordering. This is perhaps not surprising because J-band wavelengths are not far from the optical wavelengths at which the ordering was established and thus sample similar atmospheric physics. Looking individually at the $\mathrm{H}$ - and K-band spectra of Figure 3, we see larger discrepancies in the ordering. Specifically, the shape of the H-band peak near $1.65 \mu \mathrm{m}$ does not change smoothly from L2 through L8. The slope (color) of the spectrum from J through $\mathrm{K}$ also varies non-monotonically, with the spectrum of the L6 not fitting the 

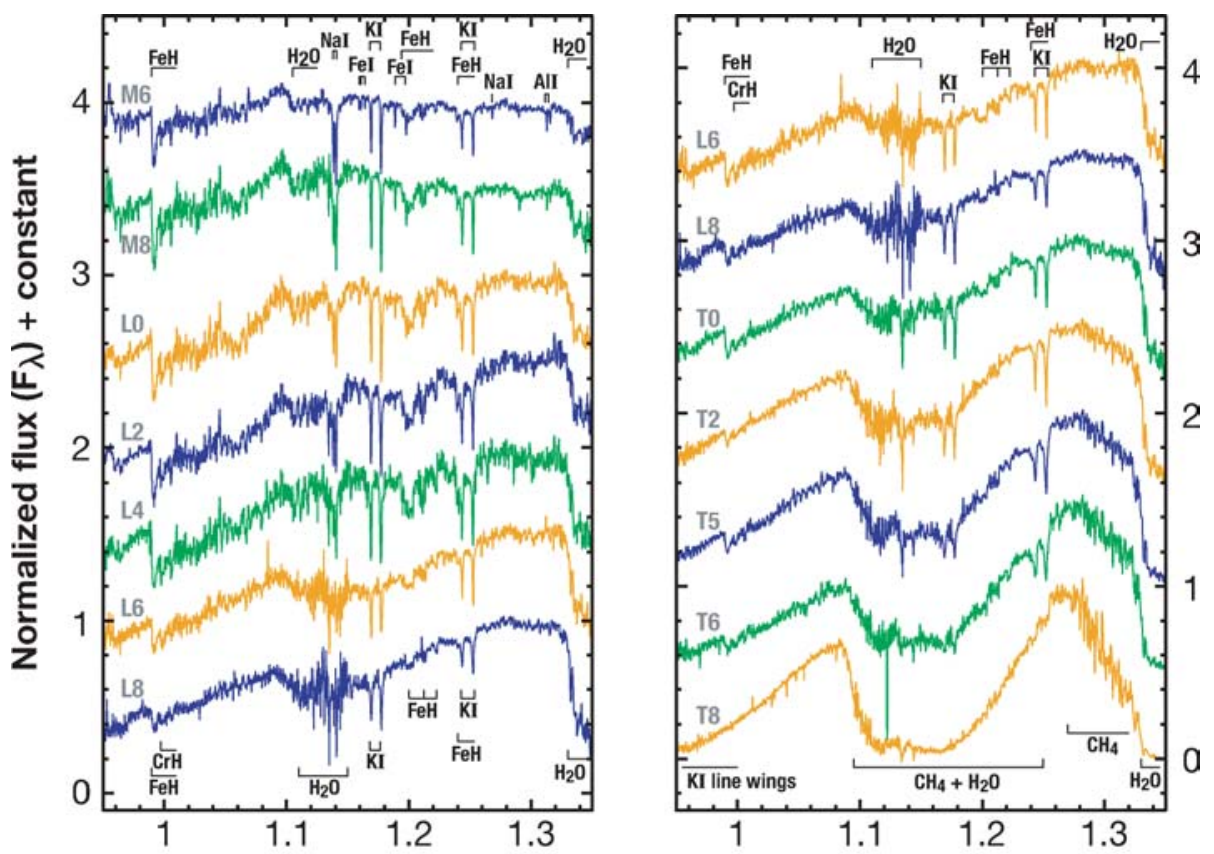

Wavelength $(\mu \mathrm{m})$

Figure 4 A $0.95-1.35 \mu \mathrm{m}$ detail of the mid-M through late-T spectral sequence shown in Figure 3. Feature identifications are marked.

trends suggested by spectra just above and below. The $\mathrm{H}$ - and $\mathrm{K}$-band spectra, being further removed from the optical, may be sampling different physical conditions in the atmosphere, or other effects such as unresolved binarity may be more easily noticeable here. The point is simply that there is a lot of information available in the JHK spectra of these objects, and in particular, care must be taken to assure that this information is properly instilled in an L dwarf near-infrared classification system.

Classification is merely a tool but a very useful one. If most objects have optical and near-infrared types that are identical with a handful of others having discordant types, it should not be interpreted that either the optical or near-infrared type of the discordant objects is wrong. Instead, this should be viewed as a clue to the atmospheric physics of those objects - that is, something about those objects make them different from the majority. Part of the power of spectral classification is its ability to flag such differences for further study. (See Section 5 for more discussion on this point.)

Compilations of near-infrared spectra of $\mathrm{L}$ and $\mathrm{T}$ dwarfs can be found in Geballe et al. (2002), Burgasser et al. (2002a), McLean et al. (2003), and Knapp et al. (2004), and in Sandy Leggett's archive at http://www.jach.hawaii.edu/ sk1/LTdata.html, and the Brown Dwarf Spectroscopic Survey archive at http://www.astro.ucla.edu/ mclean/BDSSarchive/, and at http://DwarfArchives.org. 


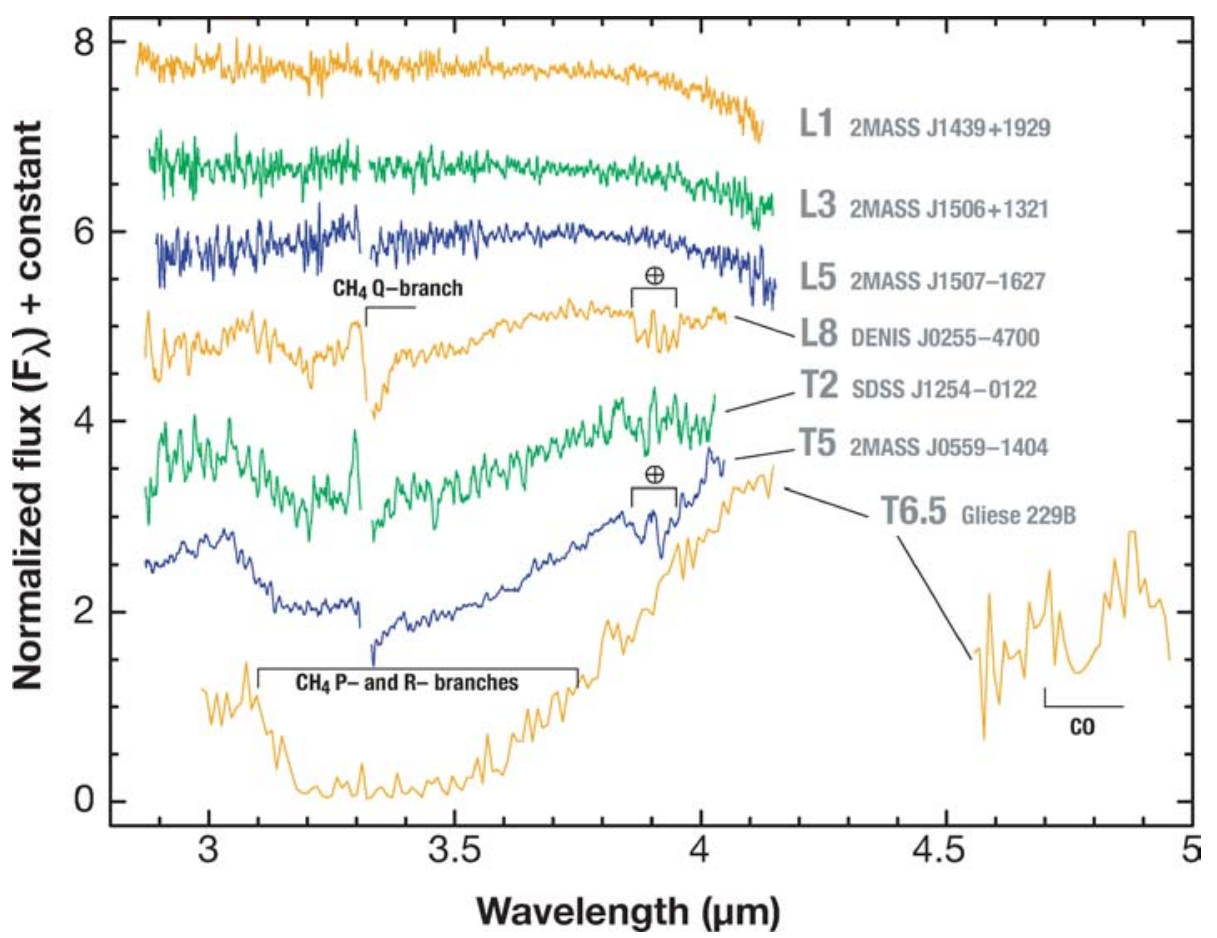

Figure 5 A sequence of 2.9 to $4.1 \mu \mathrm{m}$ spectra of early-L through mid-T dwarfs supplemented with a mid-T spectrum between 4.5 and $5.0 \mu \mathrm{m}$. The Q-, P-, and R-branches of $\mathrm{CH}_{4}$ in the $3.3 \mu \mathrm{m}$ region and the $\mathrm{CO}$ band at $4.7 \mu \mathrm{m}$ are marked. All spectra come from Cushing, Rayner, \& Vacca (2004) except for the L- and M-band spectra of G1 229B, which come from Oppenheimer et al. (1998).

\subsection{Beyond 2.5 Microns}

Independent classification at wavelengths longward of $2.5 \mu \mathrm{m}$ is not currently feasible owing to the difficulty of securing spectra there, and as a result only a few objects have been acquired at these wavelengths. Figure 5 shows L-band spectra of a sequence of L and T dwarfs from Cushing, Rayner \& Vacca (2005) and Oppenheimer et al. (1998). This sequence shows the onset of the $\mathrm{CH}_{4}$ fundamental band at $3.3 \mu \mathrm{m}$, as first noted by Noll et al. (2000). The Q-branch of the $v_{3}$ transition at $3.3 \mu \mathrm{m}$ first appears around mid-L. By late-L the P- and R-branches of $\mathrm{CH}_{4}$ also appear. By mid-T, methane has created a huge absorption trough from 3.1 to $4.0 \mu \mathrm{m}$.

Figure 5 also shows the M-band spectrum of Gl 229B from Oppenheimer et al. (1998). This spectral piece contains the fundamental band of CO at $4.7 \mu \mathrm{m}$. In Gl $229 \mathrm{~B}$, most of the flux in the 3-5 $\mu \mathrm{m}$ region is concentrated to an opacity hole around $4.1 \mu \mathrm{m}$ between these strong absorptions by $\mathrm{CH}_{4}$ at $3.3 \mu \mathrm{m}$ and $\mathrm{CO}$ at $4.7 \mu \mathrm{m}$. 


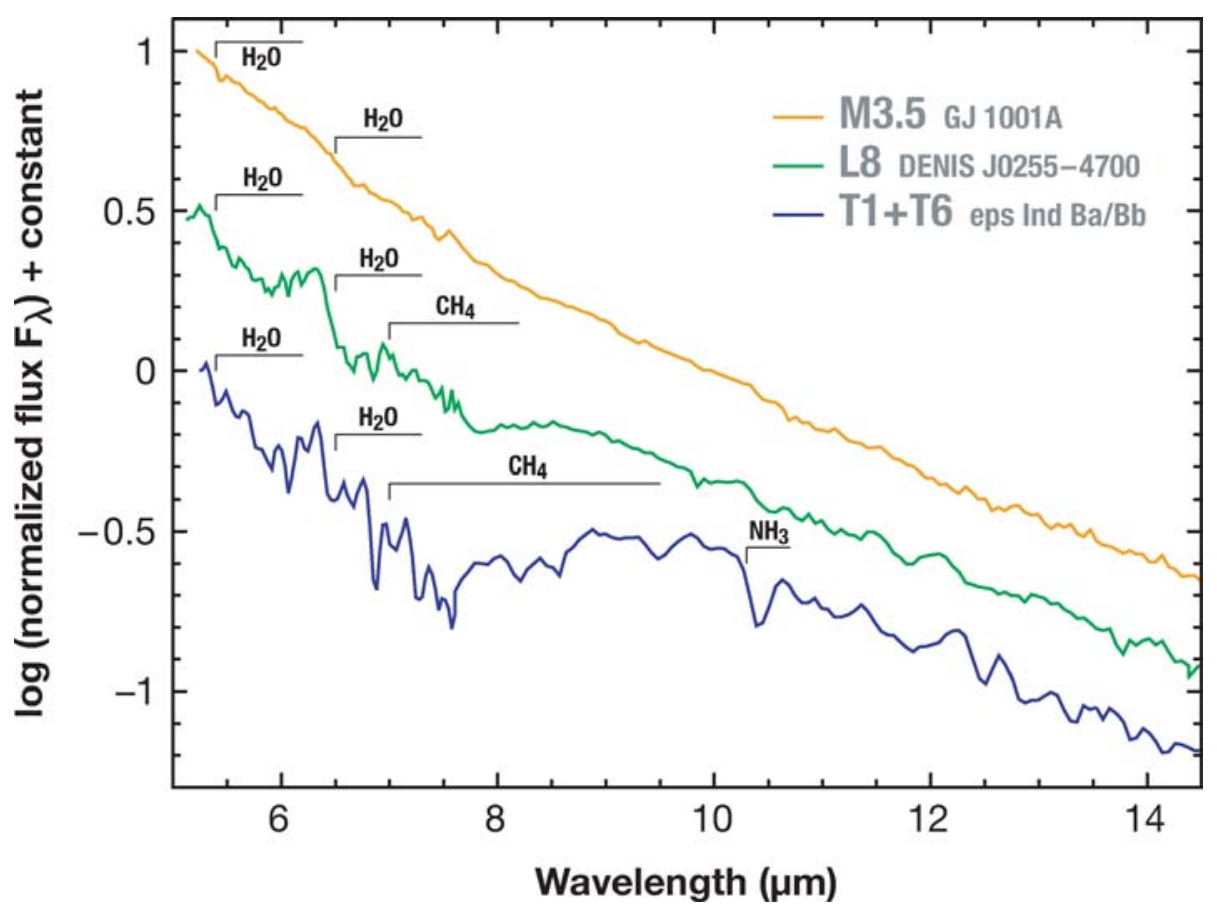

Figure 6 Spitzer IRS spectra between 5 and $15 \mu \mathrm{m}$ of an M, an L, and a T dwarf. Features caused by $\mathrm{H}_{2} \mathrm{O}, \mathrm{CH}_{4}$, and $\mathrm{NH}_{3}$ are marked. Data are taken from Roellig et al. (2004).

The launch of the Spitzer Space Telescope has enabled observations of L and T dwarfs at wavelengths longward of $5 \mu \mathrm{m}$. Figure 6 illustrates 5-15 $\mu \mathrm{m}$ spectra from Roellig et al. (2004) of an M dwarf, an L dwarf, and a T dwarf. In this region the spectrum of the $\mathrm{M}$ dwarf is dominated by absorption from $\mathrm{H}_{2} \mathrm{O}$, most obviously at 5.5 and $6.5 \mu \mathrm{m}$ but present at longer wavelengths as well. By late-L, absorption by $\mathrm{H}_{2} \mathrm{O}$ has grown stronger and $\mathrm{CH}_{4}$ centered near $7.8 \mu \mathrm{m}$ has made its appearance. In the $\mathrm{T}$ dwarf spectrum, both $\mathrm{H}_{2} \mathrm{O}$ and $\mathrm{CH}_{4}$ have strengthened and absorption by $\mathrm{NH}_{3}$ is seen between 10 and $11 \mu \mathrm{m}$. Roellig et al. (2004) also present spectra of their M and L dwarf between 15 and $35 \mu \mathrm{m}$ (not shown here), but the continua are quite featureless.

\section{THE PHYSICS UNDERLYING THE SPECTRA}

\subsection{Is the Spectral Sequence a Temperature Sequence?}

Once the empirical categorization of the spectral energy distributions is in place, researchers can begin to deduce the physics responsible for the ordering of the 
spectral sequence. For main sequence stars the most important parameter governing this ordering is temperature. It is reasonable, then, to think the same is true for $\mathrm{L}$ and $\mathrm{T}$ dwarfs. With concentrated follow-up on a subset of $\mathrm{L}$ and $\mathrm{T}$ dwarfs, we can measure temperatures semi-empirically and test this hypothesis.

If the bolometric luminosity and radius are known, the effective temperature can be deduced from the Stefan-Boltzmann law $L=4 \pi R^{2} \sigma T^{4}$. At the time of writing, no $\mathrm{L}$ and $\mathrm{T}$ dwarfs are known in eclipsing systems, so direct measurements of radii are not yet possible. Fortuitously, however, interior models indicate that field-age L and T dwarfs should have very similar radii; Monte Carlo simulations using the Burrows et al. (1997) evolutionary models for L and T dwarfs with $1 \mathrm{Myr}<$ age $<1$ Gyr and $1 M_{\text {Jup }}<$ mass $<100 M_{\text {Jup }}$ show a total range in radius of $\sim 0.90 \pm 0.15 R_{J u p}$, assuming a constant birthrate and a mass function of the form $d N \propto M^{-1} d M$ (Burgasser 2001). Thus, to first order $\mathrm{T}_{e f f}$ is directly proportional to the fourth root of luminosity. If the luminosities of many $\mathrm{L}$ and $\mathrm{T}$ dwarfs can be measured, we can determine whether the spectral sequence corresponds to a sequence in $\mathrm{T}_{\text {eff }}$ as holds for objects of type $\mathrm{O}$ through $\mathrm{M}$.

To measure luminosity, both the distance and the apparent flux summed over all wavelengths must be known. The distance can be readily computed from trigonometric parallax, but the apparent bolometric luminosity is usually deduced through a combination of empirical spectra and photometry supplemented where needed by spectral models (the latter needed just to total the generally small contribution by the short- and long-wavelength tails of the flux distribution).

For $\mathrm{L}$ and $\mathrm{T}$ dwarfs in the field, trigonometric parallaxes have been obtained by Dahn et al. (2002), Tinney et al. (2003), Thorstensen \& Kirkpatrick (2003), and Vrba et al. (2004). In addition, the distances to several L and T dwarf companions have been deduced from common proper motion with an object of higher luminosity whose trigonometric parallax has also been measured. Table 3 gives a list of the $\mathrm{L}$ and $\mathrm{T}$ dwarf parallaxes measured to date along with apparent and absolute J-band magnitudes.

Early estimates of bolometric corrections at $J$ - or $K$-band as a function of color (such as $I-K$ ) have been published by Reid et al. (2001) and Leggett et al. (2001, 2002), but the most comprehensive attempt so far is that by Golimowski et al. (2004). In this latter paper $51 \mathrm{M}, \mathrm{L}$, and T dwarfs with flux-calibrated $0.8-2.5 \mu \mathrm{m}$ spectra, $L^{\prime}$ photometry (or, for nine objects, $L^{\prime}$ estimates), and trigonometric parallaxes were used to estimate bolometric corrections at $K$-band. ${ }^{2}$ Table 3 gives the resulting "best guess" values of $T_{\text {eff }}$ as estimated by Vrba et al. (2004) alongside similar estimates by Dahn et al. (2002) and Golimowski et al. (2004). Although the uncertainties of the $T_{\text {eff }}$ measurements are generally $\pm 150-200 \mathrm{~K}$, the agreement among the three groups is very good.

\footnotetext{
${ }^{2}$ The $B C_{K}$ values from Golimowski et al. (2004) are given as a function of spectral type rather than color (like $I-J$ or $I-K$ ), which creates a dependence between temperature and type. However, as the derived values of $T_{\text {eff }}$ depend only on the fourth root of the luminosity, this dependence should be negligible.
} 


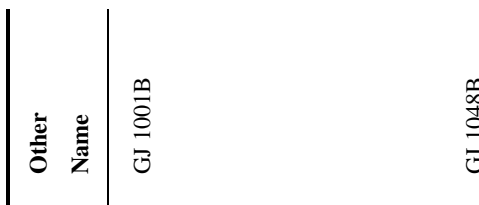

$$
\underset{\substack{\infty \\ 0}}{\stackrel{\infty}{0}}
$$

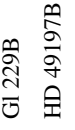

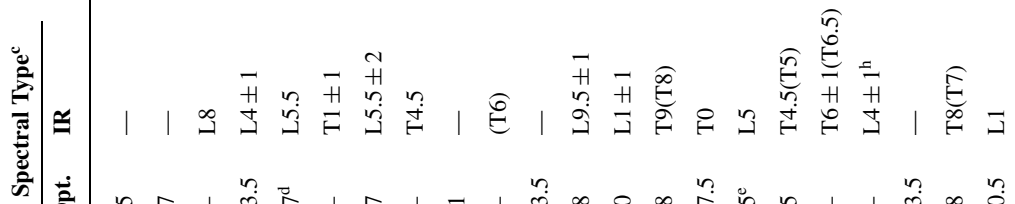
|

క్రి

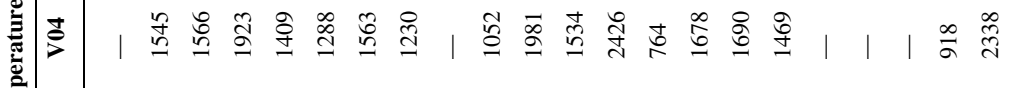
矛过|

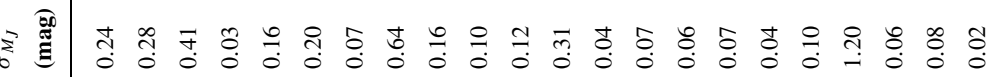

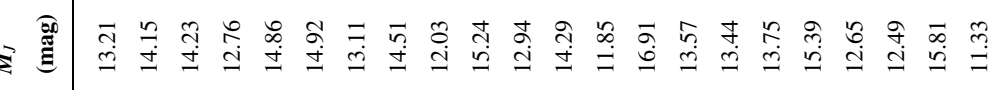




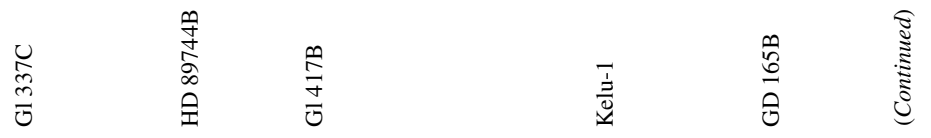

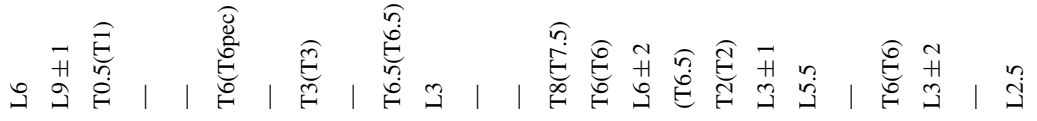

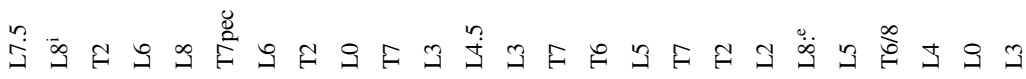

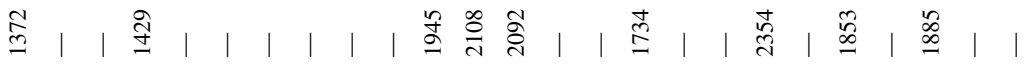

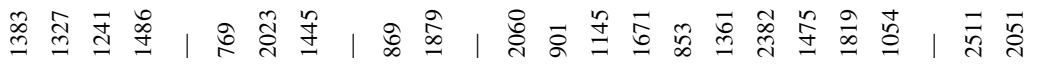

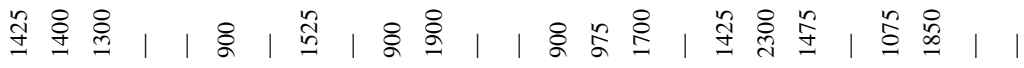

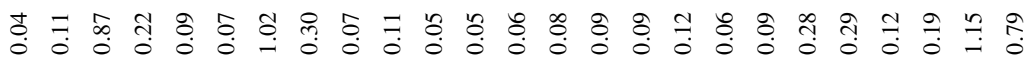

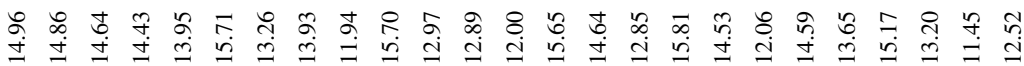
ڤ人

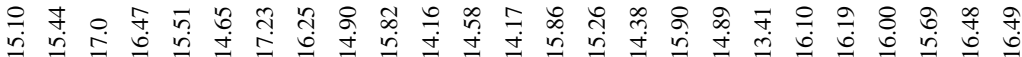

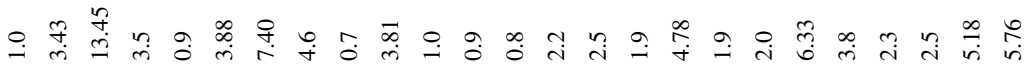

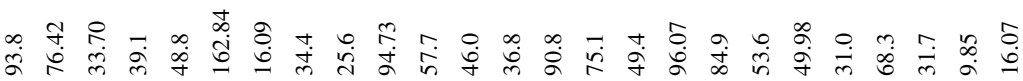

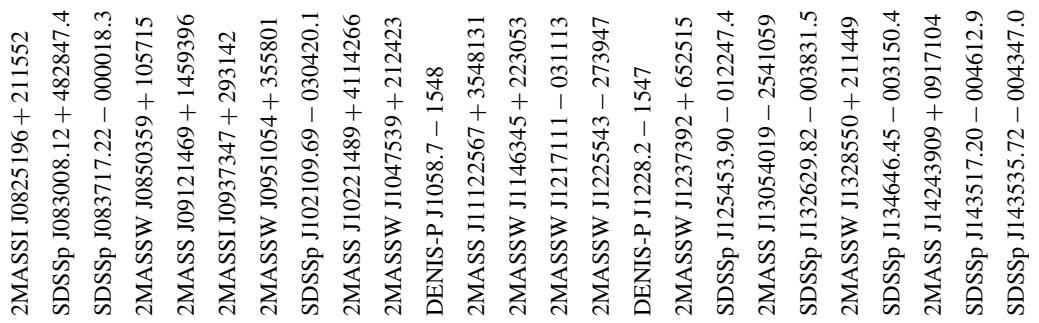




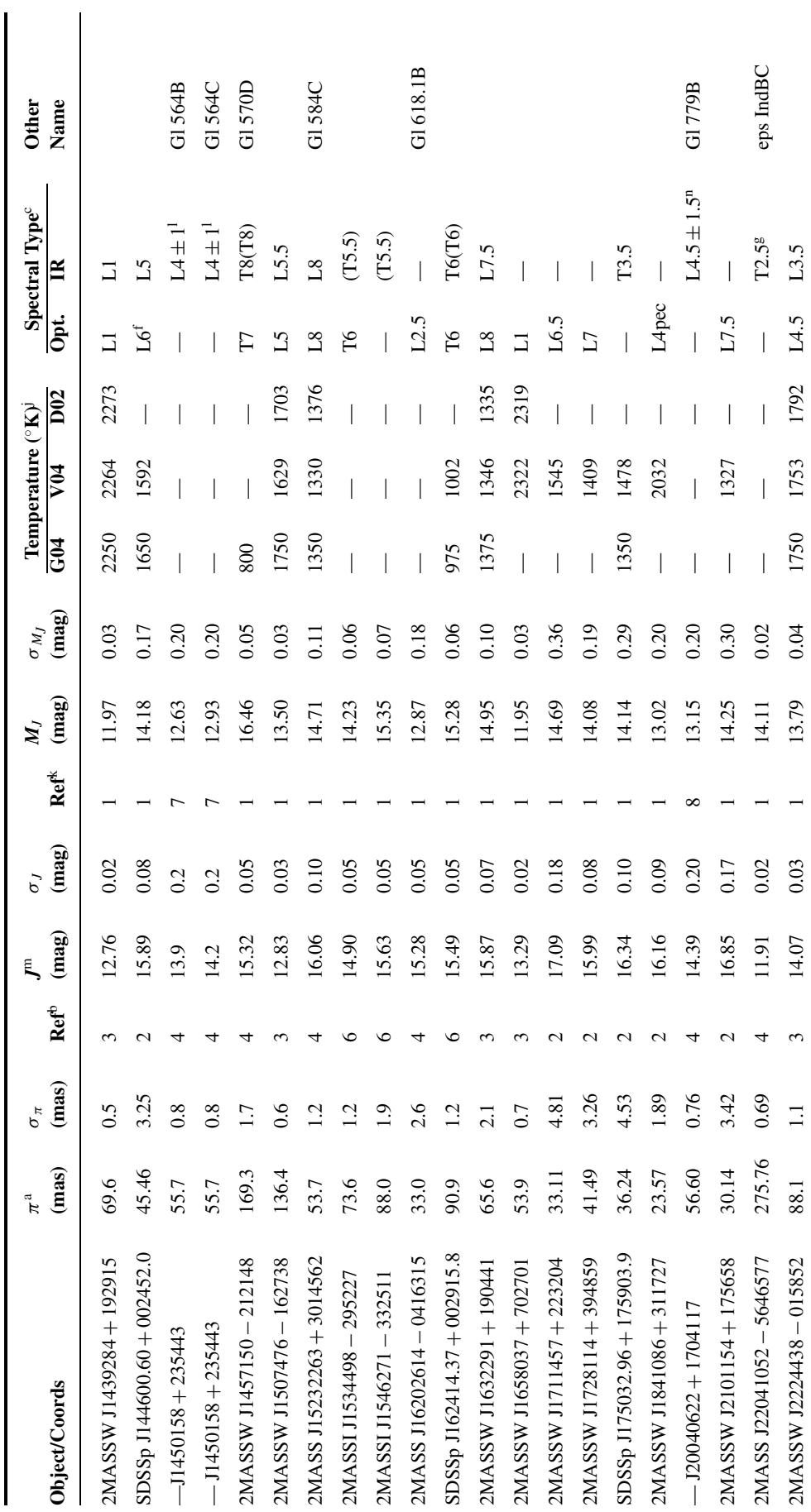




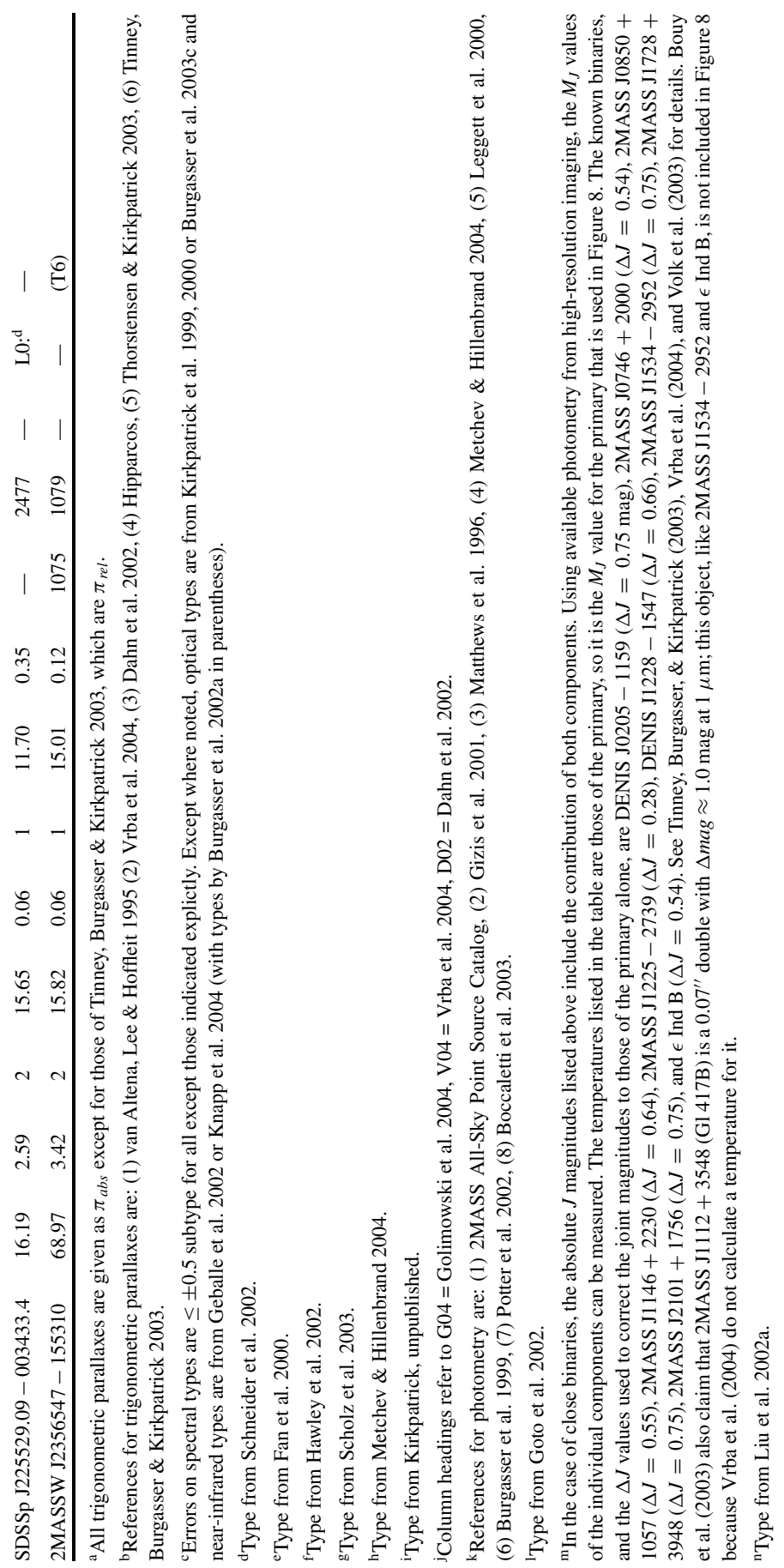


The $T_{\text {eff }}$ values from Vrba et al. (2004) along with their quoted uncertainties are plotted in Figure 7 along with the spectral types listed in Table 3. Optical type and temperature (top panel) show a tight, monotonic correlation throughout the range of $\mathrm{L}$ dwarfs but that correlation is broken at early-T. There are too few early- T dwarfs presently known to gauge the behavior accurately, but the temperatures of the T2 and T5 dwarfs appear no different from those of the latest L dwarfs. At types of T6 and later, however, the trend of cooler temperatures with later spectral type continues.

Near-infrared type shows a different behavior, as seen in the bottom panel of Figure 7. In this case temperature and spectral type are well correlated only from early- to mid-L. From mid-L to mid-T, all objects have roughly the same temperature $(\sim 1400 \mathrm{~K})$ but the scatter is large $( \pm 200 \mathrm{~K})$. At types of T6 and below, the correlation of cooler temperature with later type re-emerges.

In general, the optical type seems to be a better proxy for temperature than the near-infrared type, although there are hiccups at early-T in both sequences. Stephens (2003) noted the discrepancies between optical and near-infrared types for L dwarfs and suggested that whereas optical type was primarily a proxy for temperature, near-infrared type is more influenced by clouds or possibly gravity. Clouds, as we shall see below, are a product of condensation and sedimentation, and their presence has the effect of both veiling features in the spectra and reddening the near-infrared colors. Under both classification schemes some parameter other than temperature also seems to be playing the dominant role in shaping the spectra of objects from early- to mid-T. In this case the culprit is less obvious but, as further discussed in following sections, is likely related to the inhomogeneity of clouds and/or the disappearance of clouds below the photosphere. From mid- to late-T, the spectral type on both schemes appears again to be a good proxy for temperature.

So the question posed by the header of this section can be answered with a highly qualified "yes." The spectral sequence is a temperature sequence over part of the range, except from mid-/late-L to mid-T where cloud physics becomes as important as temperature in determining the form of the spectral energy distribution.

\subsection{Results from Chemistry Models and Synthetic Spectra}

The complex spectra of $\mathrm{L}$ and $\mathrm{T}$ dwarfs would be extremely difficult to predict a priori, but with the wealth of empirical data serving as a guide, model calculations have advanced rapidly in the past few years. Some of the most important processes governing atmospheres at $T_{\text {eff }}>700 \mathrm{~K}$ are at least partially understood.

The simplified chemical description is this: As the gas temperature of a (brown) dwarf drops, its atoms first favor an ionized state, then favor a neutral state, then begin to form molecular compounds, and finally form into a solid or liquid condensate. The exact sequence of molecules and condensates that are formed depend upon the gas pressure, the metallicity, and other factors such as mixing from warmer or colder layers. In the next two sections the physics giving rise to the spectra of field $\mathrm{L}$ and $\mathrm{T}$ dwarfs is described. 

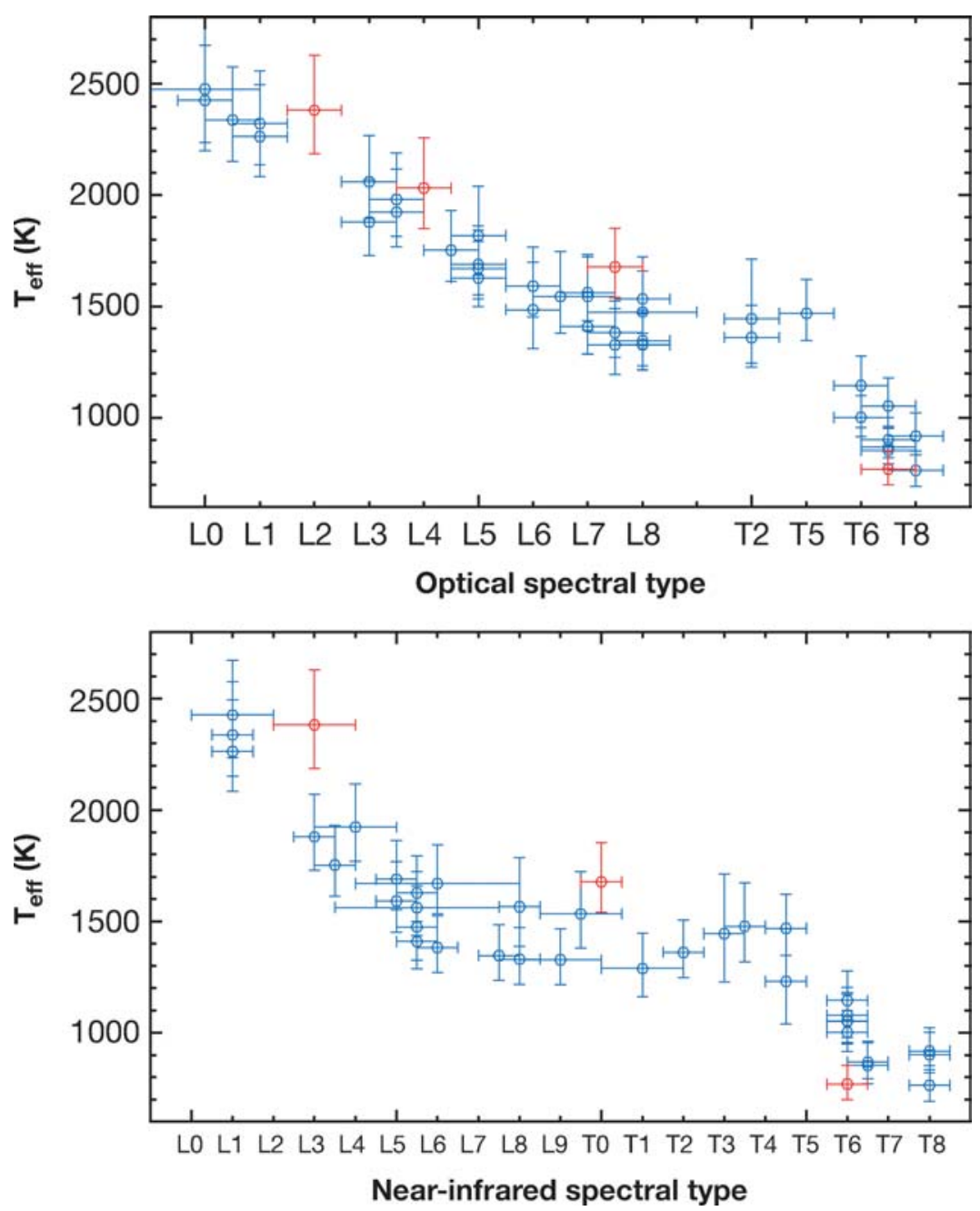

Figure 7 Effective temperature plotted against spectral type, as listed in Table 3. The top panel shows the relation between temperature and optical spectral type; the bottom panel shows temperature versus near-infrared type. Not plotted are the four objects in Table 3 with trigonometric parallax errors exceeding $30 \%$ of the parallax value itself. In the top panel, a "missing" type has been inserted between optical types of L8 and $\mathrm{T} 2$ to reflect the gap (see Section 3.1) seen in the spectral sequence there. The points in red represent four objects known to have spectroscopic peculiarities-Kelu-1 at L2-3 (near-infrared peculiarities noted by McGovern, priv. comm.), 2MASSW J1841086 + 311727 at L4 (odd optical spectrum noted by Kirkpatrick et al. 2000; near-infrared type is unknown), SDSS J0423 - 0414 at L7.5-T0 (optical/near-infrared mismatch noted by Cruz et al. 2003; duplicity noted by Burgasser, priv. comm.), and 2MASS J0937+ 2931 at T6-T7 (spectroscopic peculiarity noted by Burgasser et al. 2002a). 
4.2.1. L DWARFS As noted in Figure 2, early-L dwarfs are noted for the disappearance of $\mathrm{TiO}$ and $\mathrm{VO}$, molecules whose prominent optical bands are the hallmarks of M-type spectra. Chemical equilibrium calculations (e.g., Lodders 1999, Burrows \& Sharp 1999, Allard et al. 2001) suggest that TiO disappears from the spectra because it converts into $\mathrm{TiO}_{2}$ or condenses into perovskite $\left(\mathrm{CaTiO}_{3}\right)$ and other titanium-bearing molecules $\left(\mathrm{Ca}_{4} \mathrm{Ti}_{3} \mathrm{O}_{10}, \mathrm{Ca}_{3} \mathrm{Ti}_{2} \mathrm{O}_{7}, \mathrm{Ti}_{2} \mathrm{O}_{3}, \mathrm{Ti}_{3} \mathrm{O}_{5}\right.$, and $\left.\mathrm{Ti}_{4} \mathrm{O}_{7}\right)$ at temperatures near the $\mathrm{M} / \mathrm{L}$ boundary. Vanadium is less refractory than titanium, so $\mathrm{VO}$ is removed from the gas at slightly lower temperatures than the removal of TiO. Lodders (2002) argues that the $\mathrm{VO}$ is removed via formation of $\mathrm{VO}_{2}$ and a condensate not of pure VO as others have stated, but instead as a solid solution with titanium-bearing condensates.

The condensates mentioned above have a very noticeable effect on the emergent spectrum because they clear the photosphere of two of its main absorbers. Other condensates have a much less obvious, but no less important, effect. Aluminumbearing condensates such as corundum $\left(\mathrm{Al}_{2} \mathrm{O}_{3}\right)$ and calcium aluminates like hibonite $\left(\mathrm{CaAl}_{12} \mathrm{O}_{19}\right)$, grossite $\left(\mathrm{CaAl}_{4} \mathrm{O}_{7}\right)$, and gehlenite $\left(\mathrm{Ca}_{2} \mathrm{Al}_{2} \mathrm{SiO}_{7}\right)$ form at even higher temperatures than the titanium-bearing condensates mentioned above. However, these first condensates remove calcium that might otherwise go toward perovskite formation, and this has the direct effect of determining the rate at which the atmosphere is robbed of its TiO. Likewise, forsterite $\left(\mathrm{Mg}_{2} \mathrm{SiO}_{4}\right)$ and enstatite $\left(\mathrm{MgSiO}_{3}\right)$, which are somewhat less refractory than the $\mathrm{Ca}$ and $\mathrm{Al}$ silicates and thus form at slightly cooler temperatures, sequester some of the oxygen and prohibit it from forming other compounds at cooler temperatures. If elements such as $\mathrm{Al}, \mathrm{Ca}$, and $\mathrm{Si}$ were not lost via such condensate-cloud formation at higher temperatures, neutral potassium would not have been seen as a major absorber in late- $\mathrm{L}$ and $\mathrm{T}$ dwarfs because it would have been removed by silicate condensates like orthoclase ( $\mathrm{KAlSi}_{3} \mathrm{O}_{8}$, sometimes referred to as high sanidine). Such is the bizarre and intricate dance of the condensates in $\mathrm{M}, \mathrm{L}$, and T dwarf atmospheres. For more information on this topic, the reader is referred to the detailed papers by Lodders (2002) and Lodders \& Fegley (2002) and the review by Burrows et al. (2001).

As the Ti-bearing condensates form at late-M and early-L, the opacity of the oxide bands weakens and the contrast of the alkali lines and hydride bands against the relative continuum is increased. By mid- to late-L the ground state neutral lines of $\mathrm{Na} \mathrm{I}$ and $\mathrm{K} \mathrm{I}$ are the dominant absorbers in the optical, their wings covering thousands of $\AA$. Burrows \& Volobuyev (2003) point out that the rainout of condensates clears the atmosphere of most of its metals and leaves the less refractory neutral alkali metals as the only major sources of opacity between 4000 and 10,000 $\AA$. Sodium and potassium are the most abundant of the alkali metals at solar metallicity (Anders \& Grevesse $1989^{3}$ ) and so have the most influence

\footnotetext{
${ }^{3}$ Tsuji (2000) notes that the Anders \& Grevesse (1989) values may need revision for other elements because there are new solar abundance measures of C from Grevesse et al. (1991), $\mathrm{N}$ from Grevesse et al. (1990), O from Allende Prieto, Lambert, \& Asplund (2001), and Fe from Biemont et al. (1991) and Holweger et al. (1991).
} 
on the shape of the spectral energy distribution in the optical. These $\mathrm{Na}$ and $\mathrm{K}$ atoms have their energy levels perturbed by the potential field of $\mathrm{H}_{2}$, which is the dominant molecule in a gas at $\mathrm{L}$ and $\mathrm{T}$ dwarf temperatures. Burrows \& Volobuyev (2003) studied the absorption cross-sections of $\mathrm{H}_{2}$-perturbed sodium and potassium atoms using quantum chemical codes and were able to model the red wing of the K I doublet $(7665,7699 \AA)$ and the red wing of the Na I doublet (5890, $5896 \AA$ ). They found that the influence of K I on the continuum shape extends out to $9500-10,000 \AA$, and the wings of Na I extend out to $\sim 8000 \AA$. These results explain the shape of the optical continua of mid-L to T dwarfs as shown in Figure 1. Allard et al. (2003), on the other hand, argue that these broad alkali wings still do not account for all of the opacity in the optical and that another mystery absorber is still present.

At early-L through early- $\mathrm{T}$, absorptions by hydride molecules are also seen in the optical and near-infrared. The empirical spectra at early- to mid-L (Figures 2 and 4) show the strength of first $\mathrm{FeH}$ then $\mathrm{CrH}$ reaching a peak, with both beginning to weaken and disappear around early-T. Chemical equilibrium calculations imply that $\mathrm{CrH}$ should remain at temperatures cooler than the disappearance of $\mathrm{FeH}$ (Burrows et al. 2001). However, a robust calculation has not yet been made because sufficiently complete line lists for both molecules have only recently been published by Burrows et al. (2002) for $\mathrm{CrH}$ and by Dulick et al. (2003; see also Cushing et al. 2003) for FeH. This piece of the atmospheric chemistry puzzle still must be addressed thoroughly. One curious observation about $\mathrm{FeH}$ was noted by Burgasser et al. (2002b), who showed that the Wing-Ford (9896 ̊) band weakens substantially in the spectral sequence by late-L but then regains strength at early-T before disappearing entirely by mid-T. The physics of the $\mathrm{L} / \mathrm{T}$ transition is discussed further in the next section.

At mid-L the methane fundamental band at $3.3 \mu \mathrm{m}$ is first seen (Noll et al. 2000; Cushing, Rayner, \& Vacca 2004), indicating that the conversion of carbon monoxide to methane as the dominant carbon-bearing molecule via the reaction $\mathrm{CO}+3 \mathrm{H}_{2} \rightarrow \mathrm{CH}_{4}+\mathrm{H}_{2} \mathrm{O}$ has begun. This reaction produces more $\mathrm{H}_{2} \mathrm{O}$, so water bands also continue to strengthen. Methane is also seen at $7.8 \mu \mathrm{m}$ in late- $\mathrm{L}$ dwarfs, which is not surprising as this band should have a strength comparable to that of the $3.3 \mu \mathrm{m}$ feature (Roellig et al. 2004). The overtone bands of methane at 1.6 and $2.2 \mu \mathrm{m}$ are also sometimes seen in late-L dwarfs (e.g., McLean et al. 2001; Nakajima, Tsuji, \& Yanagisawa 2004) but are not clearly detected at low resolution (i.e., resolving powers of $\mathrm{R}=\lambda / \Delta \lambda<2000$ ) until type T0. This unambiguous detection of $\mathrm{CH}_{4}$ at $\mathrm{H}$ - and $\mathrm{K}$-bands is the defining characteristic of $\mathrm{T}$ dwarfs.

\subsubsection{T DWARFS}

4.2.2.1. The effects of condensates The condensates mentioned in the last section have an even more dominant effect in shaping spectra from early- to mid-T. The vertical distribution of these condensates, their number, and their size all play an important role in determining the extent to which grain scattering and absorption 
control the emergent flux (Burrows et al. 2001). Spectral modeling has shown that the magnitudes and very red near-infrared colors of the latest $\mathrm{L}$ dwarfs $\left(J-K_{S}\right.$ $\approx 2$; see Figure 8 ) cannot be explained without invoking these condensates as a dusty component of the photosphere (e.g., the case B models of Tsuji 2000 and the AMES-dusty models of Allard et al. 2001), but these same models completely fail to explain the colors of T dwarfs. Models in which the dust has been allowed to disappear from the photosphere because of gravitational settling (e.g., the case C models of Tsuji 2000 and the AMES-cond models of Allard et al. 2001) can predict the colors $\left(J-K_{s} \approx 0\right)$ and magnitudes of the mid- to late-T dwarfs, but fail to explain the behavior of the early-T dwarfs. The trick is explaining what happens in the atmospheres of objects in the early- $T$ dwarf regime where presumably the settling of condensates is the most important physical process.

Tsuji (2002) discusses the temperature range between the beginning of dust condensation $\left(T_{\text {cond }}\right)$ and the critical point at which the dust grains grow too large to remain suspended in the photosphere $\left(T_{c r}\right)$. This critical temperature cannot presently be modeled but can be constrained by the observed spectra. In the Tsuji models, the dust cloud is located within the optically thin region of the photosphere for $T_{\text {eff }}>1600 \mathrm{~K}$. At the lowest temperatures in this range, dust extinction veils the molecular line strengths considerably. In the range $1500 \mathrm{~K}<T_{\text {eff }}<1700 \mathrm{~K}$ the dust cloud thickens even more but also begins to fall into the optically thick regime where it is a less important contributor to the emergent flux. These two effects tend to cancel one another so that there is little change in the column density of dust in the observable photosphere. At $T_{\text {eff }}<1400 \mathrm{~K}$ the dust moves deeper into the atmosphere, allowing the upper atmosphere to cool rapidly, triggering the onset of $\mathrm{CO}$ to $\mathrm{CH}_{4}$ conversion and the reversal of the near-infrared colors. At face value, this appears to be an elegant description of the physical process, but there is one problem: This reversal of the near-infrared colors is too slow in the Tsuji (2002) model; that is, the $J-K$ colors move from $\sim 2$ to $\sim 0$ over a span no narrower than $\sim 600 \mathrm{~K}$ in $T_{\text {eff }}$. As Figure 8 (bottom panel) illustrates, the empirical data show that the temperature range of this color transition is only $\sim 200 \mathrm{~K}-300 \mathrm{~K}$.

Marley et al. (2002) present a similar approach to Tsuji (2002) by also considering the placement of the condensate clouds relative to the observable photosphere. In the Marley et al. (2002) models the physics of the cloud layer is governed by a parameter $f_{\text {rain }}$ (now more appropriately called $f_{\text {sed }}$; Knapp et al. 2004), which is the efficiency of sedimentation relative to the turbulent mixing. A value of $f_{\text {sed }}=0$ corresponds to a well mixed, very dusty atmosphere with no sedimentation to offset mixing; large values of $f_{\text {sed }}$ correspond to more efficient sedimentation and thinner clouds. The colors of the reddest $\mathrm{L}$ dwarfs can be explained by models with $f_{\text {sed }}<3$, whereas early-T dwarfs require $f_{\text {sed }}>3$. Mid-T and later dwarfs are best fit with cloud-free models $\left(f_{\text {sed }} \approx \infty\right)$. These results agree conceptually with other work but, like the Tsuji (2002) model, the Marley et al. (2002) models fail to reproduce the rapid turnaround of $J-K$ color from red to blue (see also figure 1 of Burgasser et al. 2002b).

Burgasser et al. (2002b), elaborating on an idea suggested by Ackerman \& Marley (2001), have used the Marley et al. (2002) models to propose a possible 


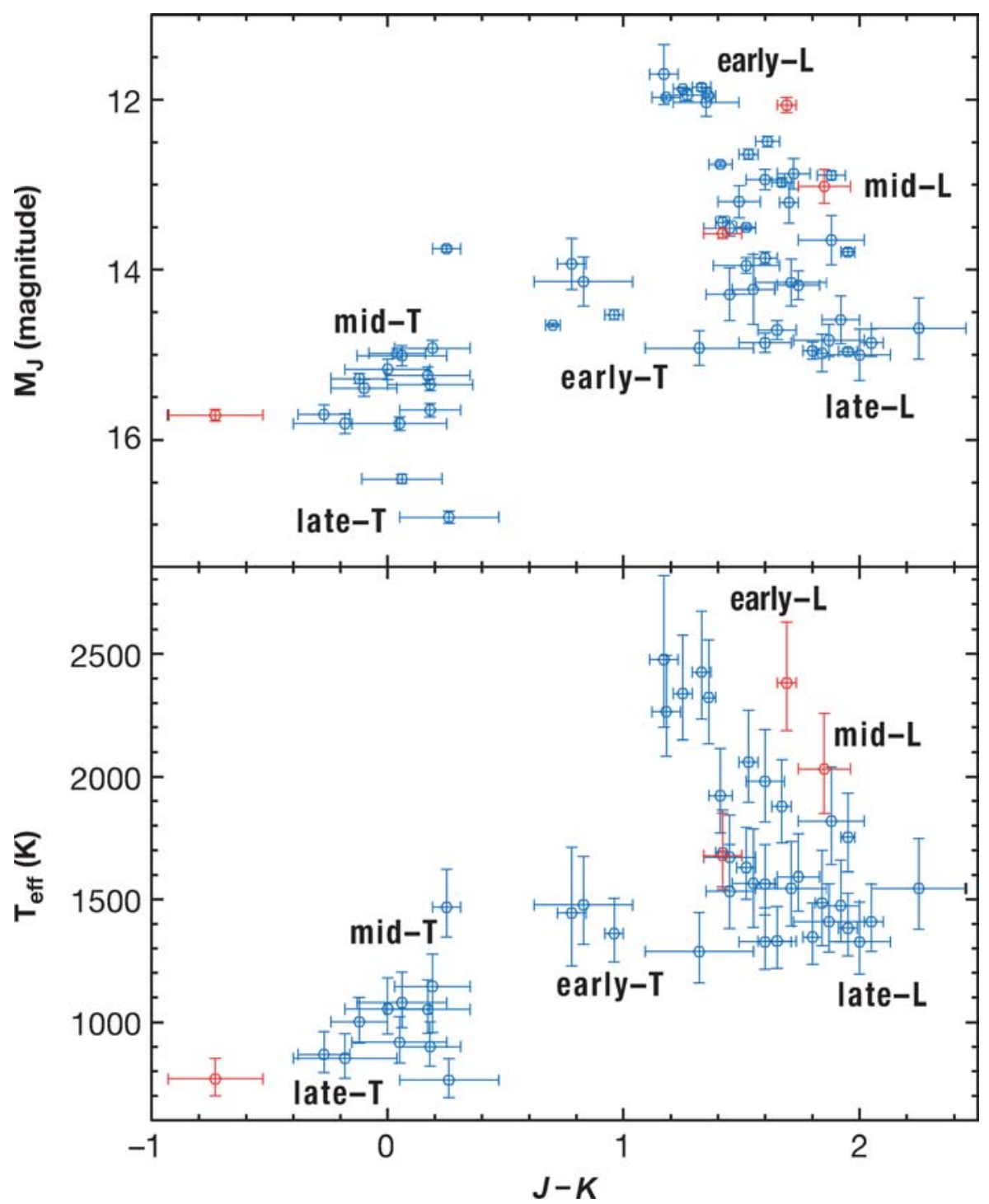

Figure 8 (Top panel) Absolute J-band magnitude versus $J-K$ color for L and T dwarfs from Table 3. (Bottom panel) Effective temperature versus $J-K$ color for the same data set. The colors come from data tabulated in Vrba et al. (2004) supplemented with data from 2MASS. As such, the colors are not strictly homogeneous (the 2MASS values are $J-K_{s}$ on the CIT system, for example), but any system-to-system color differences should be small compared to the 3.5-magnitude spread along the x-axis. Approximate locations of early- $\mathrm{L}$ through late-T dwarfs are shown in black. The four spectroscopically peculiar objects from Figure 7 are again noted in red and represent, from left to right, 2MASS J0937 + 2931, SDSS J0423 - 0414, Kelu-1, and 2MASS J1841 + 3117. 
solution. The cloudy models that adequately explain late- $\mathrm{L}$ dwarfs assume a uniform distribution of condensates over the visible disk, but Burgasser et al. (2002b) note that clouds seen in the atmospheres of Jupiter and Saturn show discrete banding and spotting. Breaks in the cloud decks of these planets allow flux from warmer layers to emerge, such as is seen in the $5 \mu \mathrm{m}$ "holes" in Jupiter's atmosphere. Under this scenario, the location of a transition object on the $M_{J}$ versus $J-$ $K_{s}$ diagram depends on its percentage of cloud coverage. Clearer atmospheres are bluer in $J-K_{S}$ and brighter in $M_{J}$ than cloudier counterparts of the same temperature. The reversal in strength of the Wing-Ford FeH band at $9896 \AA$ for early- $\mathrm{T}$ dwarfs (mentioned in Section 4.2.1) can also be explained under this scenario. In the colder layers above the clouds, the condensation of iron has robbed the atmosphere of most of its FeH-bearing gases, so little $\mathrm{FeH}$ is seen in the spectra of late-L dwarfs. At early-T, however, the opening of $1 \mu \mathrm{m}$ holes in the clouds allows light from the deeper, warmer layers to emerge, and in these warmer layers $\mathrm{FeH}$ has not been depleted. Thus FeH grows in strength again at early- to mid-T then eventually weakens again at late-T types, where the atmosphere has cooled to the point where none of the observable layers contain $\mathrm{FeH}$.

Tsuji \& Nakajima (2003) offer a different solution for the quick turn around of $J-K_{S}$ colors for early-T dwarfs. They suggest that the observational $M_{J}$ versus $J-K$ diagram should not be interpreted as a single evolutionary sequence. Rather, what we are seeing is a snapshot of objects descending along slightly different evolutionary sequences as dictated by their gravities. In this case, the Tsuji (2002) model is correct; only our previous interpretation of the observational diagram is in error. (See figure 2 of Tsuji \& Nakajima 2003.) For example, a high-gravity object would lose its cloud deck below the observable photosphere at a much dimmer value of $M_{J}$ (and cooler temperature) than a low-gravity object would. Under this model the difference in the $M_{J}$ value of the turnoff between an object of mass 70 $M_{J u p}$ and one of $10 M_{J u p}$ is $\sim 1.5$ magnitudes. On a diagram such as Figure 9, we should interpret the brightest objects in $M_{J}$ between mid-L and mid-T as being the ones of lowest gravity (i.e., lowest mass); the dimmest of the mid-L to mid-T dwarfs would be the highest gravity ones (highest mass).

Knapp et al. (2004) propose a third scenario to explain the sudden blue turn of near-infrared colors at late-L/early-T. They refer to their idea as the sudden downpour model. Under this scenario, all objects pass through late-L types (roughly the same $M_{J}$ value) before reaching the T dwarf regime. The early-T sequence in this case represents stages in a rapid rain-out of condensates from the observable photosphere. Late-L through early-T represents the onset of the downpour, and mid-T represents its conclusion. After the downpour of condensates is complete, the spectral sequence from mid-T to later types is again a cooling sequence denoting $T_{\text {eff }}$.

As explained in Knapp et al. (2004), each of the three scenarios outlined above has predictions that are testable. With the proper observational data and sufficiently large samples for statistical robustness, we will be able to judge which, if any, of these models is likely to be correct. 


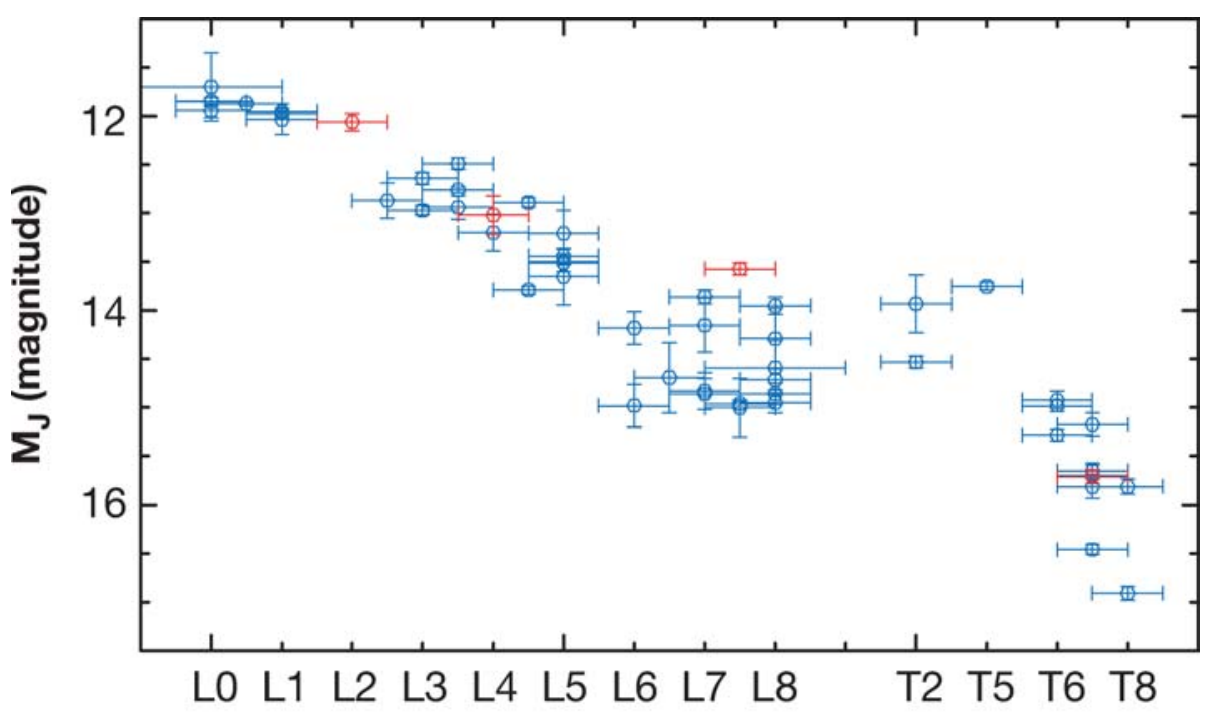

\section{Optical spectral type}

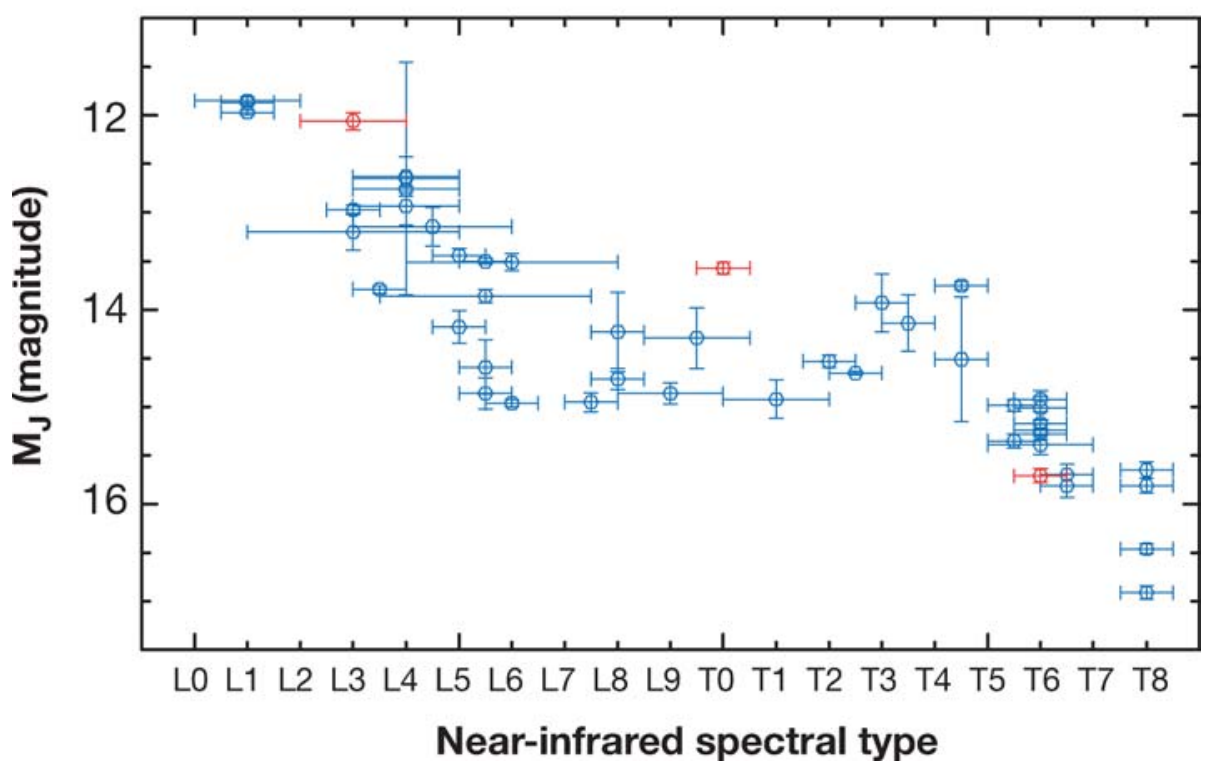

Figure 9 Absolute $J$-band magnitude versus spectral type for the optical classification system (top) and near-infrared system (bottom). The data come from Table 3, where the $M_{J}$ values for known binaries are adjusted to reflect the absolute magnitude of the primary alone. (See table footnotes for a discussion.) Not plotted are the four objects in Table 3 with trigonometric parallax errors exceeding $30 \%$ of the parallax value itself. The points in red are the same spectroscopically peculiar objects noted in Figures 7 and 8. 
4.2.2.2. Other sources of opacity Aside from the aforementioned absorption by $\mathrm{CH}_{4}$ and $\mathrm{H}_{2} \mathrm{O}$ and the dominant effect by grains, three other absorbers also become important in $\mathrm{T}$ dwarfs. The first is ammonia, $\mathrm{NH}_{3}$, which was first (possibly?) detected in the spectrum of Gl 229B at $2.0 \mu \mathrm{m}$ by Saumon et al. (2000). It is clearly detected at $10.5 \mu \mathrm{m}$ in the spectrum of $\epsilon$ Ind $\mathrm{Ba} / \mathrm{Bb}$ (Roellig et al. 2004). Atmospheric models suggest that $\mathrm{NH}_{3}$ will become more important at temperatures cooler than those of the coolest known T dwarfs, a point re-addressed in Section 6.

The second important absorber is one that is very difficult to discern empirically but is nonetheless needed to explain the observational spectra. This is absorption caused by the induced dipole moment from transient interactions by $\mathrm{H}_{2}$. At high densities of $\mathrm{L}$ and $\mathrm{T}$ dwarfs, collisions are frequent, and at such cool temperatures $\mathrm{H}_{2}$ is a very abundant molecule. The result is a nearly continuous absorption throughout the infrared spectrum (Tsuji 2002). As can be seen from figure 3 of Borysow, Jorgensen, \& Zheng (1997), it has its strongest influence in the K-band region $(\sim 2.2 \mu \mathrm{m})$. The location of this collision-induced absorption in the spectra of $\mathrm{T}$ dwarfs is marked on Figure 3.

The third absorber is CO. Based on strict models of equilibrium chemistry, the importance of $\mathrm{CO}$ should diminish greatly below the temperature at which $\mathrm{CO}$ to $\mathrm{CH}_{4}$ conversion takes place. Nonetheless, as we have seen, $\mathrm{CO}$ continues to be an important absorber in the spectrum of Gl 229B at $4.7 \mu \mathrm{m}$ as first pointed out by Noll, Geballe, \& Marley (1997). The explanation favored by those and subsequent authors is that $\mathrm{CO}$ is dredged up from warmer, lower layers into the photosphere where it is seen in the spectrum. Saumon et al. (2000) demonstrate that the observed $\mathrm{CO}$ abundance is some three orders of magnitude higher than equilibrium models would dictate. This nonequilibrium condition exists because the timescale for vertical transport of $\mathrm{CO}$ from warmer to colder layers as well as the timescale for conversion of $\mathrm{CH}_{4}$ into $\mathrm{CO}$ are faster than the conversion timescale of $\mathrm{CO}$ back into $\mathrm{CH}_{4}$ (Saumon et al. 2003).

\subsection{The Nature and Number of $\mathrm{L}$ and $\mathrm{T}$ Dwarfs}

Now that we have addressed the atmospheres of these objects in some detail, it is natural to ask about the importance of these objects in a Galactic context. What are $\mathrm{L}$ and $\mathrm{T}$ dwarfs and how do their space and mass density compare to those of other objects?

4.3.1. NATURE In Section 1 it was stated that $\mathrm{L}$ and $\mathrm{T}$ dwarfs were first uncovered during searches for brown dwarfs. Are all L and T dwarfs substellar or are some of them hydrogen-burning stars? To answer this question, we look toward theory for guidance. Interior models of low-mass stars and brown dwarfs have been constructed by Burrows et al. (1997), Chabrier et al. (2000), and Baraffe et al. (2003). We show evolutionary tracks from these models on the $T_{\text {eff }}$ versus age diagrams of Figure 10. We have selected an earlier set of models (vintage 1997, before the burst of L and T dwarf discoveries) and a later set (vintage 2000/2003, 

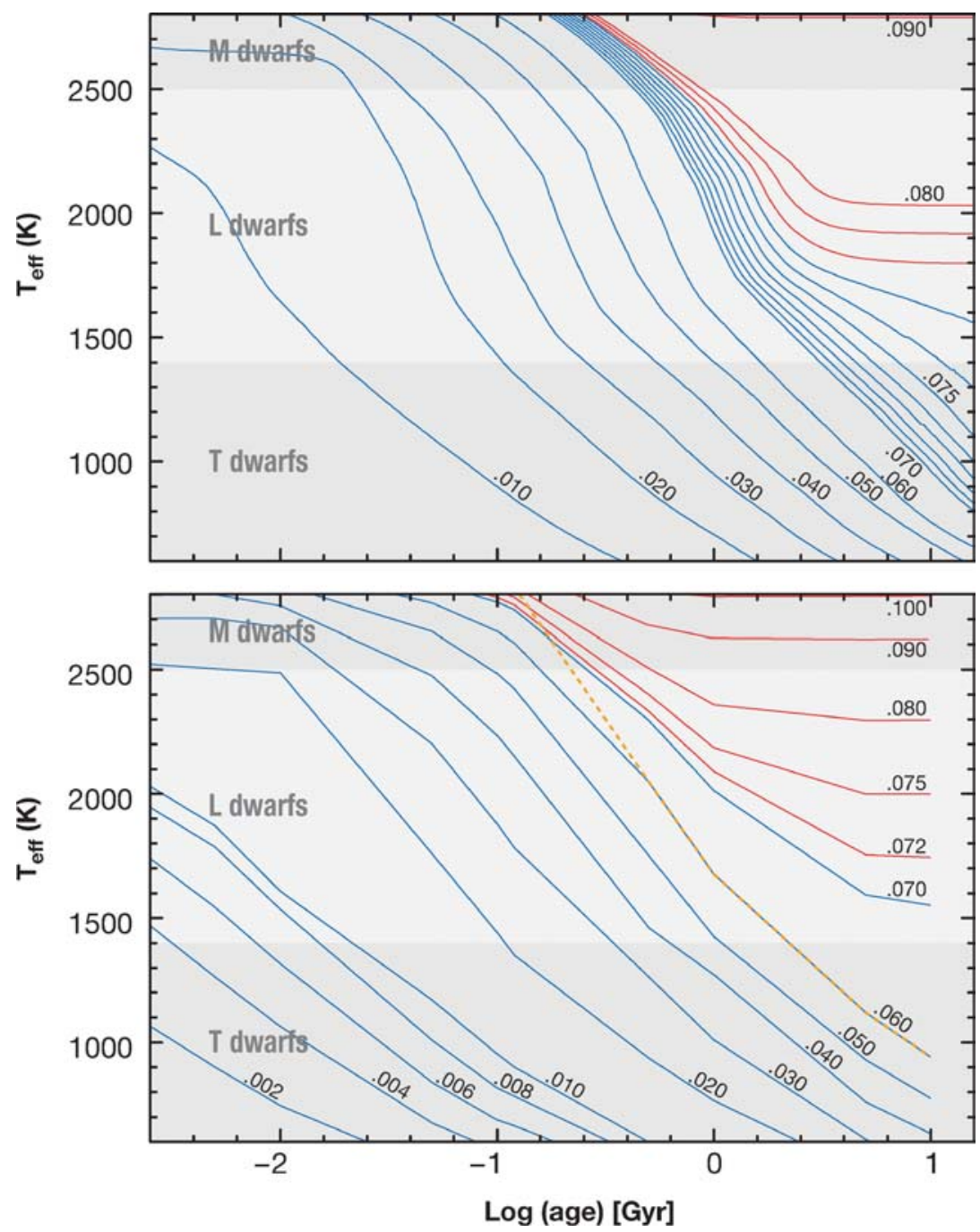

Figure 10 Theoretical tracks showing the evolution of effective temperature with age for low-mass stars (red) and brown dwarfs (blue). Grey areas delineate the approximate temperature ranges, as determined from the top panel of Figure 7, for the optical L and T dwarf sequences. The tracks come from Burrows et al. (1997) for the top panel. For the bottom panel, the tracks warmer than $1500 \mathrm{~K}$ come from Chabrier et al. (2000, using the AMES-dusty models of Allard et al. 2001) and the tracks cooler than $1500 \mathrm{~K}$ come from Baraffe et al. (2003, using the AMES-cond models of Allard et al. 2001). The line marking the boundary at which lithium is $50 \%$ depleted is shown by the orange dots in the lower panel (see Chabrier et al. 2000). Objects to the right of this orange dotted line will have burned their primordial stock of lithium; objects to the left will not have burned their lithium. See Section 4.3.1 for discussion. 
after the initial flurry of discoveries). This has been done purposely to highlight the fact that such models are still in good relative agreement with one another. This is the case despite the fact that the later models have coupled the latest atmospheric profiles (as guided by empirical spectra) with the inner structure of the stars and brown dwarfs. Another way of interpreting the agreement between old and new sets is that details of the atmospheric models have little effect on the overall evolutionary properties of the object.

In both panels of Figure 10, the tracks shown in red represent objects that (eventually) reach a long period of sustained thermonuclear fusion ${ }^{4}$ and thus settle onto the main sequence. In the models of Burrows et al. (1997, top panel) the lowest mass object that can reach this configuration is $\sim 78 \mathrm{MJup}_{\text {Jup }}$ in the models of Chabrier et al. (2000, bottom panel) and Baraffe et al. (2003), this lowest mass is $\sim 72 M_{\text {Jup }}$. These represent the lowest masses that an object can have and still be a star. (Note that these models are for solar metallicity; lower metal abundances push the main sequence minimum mass to higher masses; Chabrier \& Baraffe 1997. See Section 5.3 for further discussion.) Tracks of lower mass are shown in blue; because these objects have insufficient mass to sustain normal hydrogen burning, they are brown dwarfs by definition.

Also marked on Figure 10 are the approximate temperature ranges spanned by the optical $\mathrm{L}$ and $\mathrm{T}$ spectral classes (refer to Figure 7, top panel). Theory then suggests that early- to mid-L dwarfs are a mixture of low-mass stars $\left(<85 M_{J u p}\right)$ that are fairly old ( $>300 \mathrm{Myr}$ ) and brown dwarfs generally younger than $\sim 3 \mathrm{Gyr}$. The latest $\mathrm{L}$ dwarfs are, however, all brown dwarfs — spanning the range from very high mass ones $\left(\sim 70 M_{J u p}\right)$ that are as old as or older than the Sun to very low mass ones that are still very young. Stars do not probe down into the temperature range spanned by $\mathrm{T}$ dwarfs, however, so all $\mathrm{T}$ dwarfs are substellar.

It should be noted here that some astronomers loosely use terms such as L stars and T stars, which are often improper uses of the word "stars." Objects of type T are not believed to be stars in the traditional sense, so there can be no such thing as a $\mathrm{T}$ star. As noted above, objects of type $\mathrm{L}$ represent a mixture of stars and brown dwarfs. The term $\mathrm{L}$ star could thus be used to distinguish an object from a lower mass L brown dwarf, but in most instances, the term L star is used with no reference to the stellar or substellar nature of the object being intended. The terms L dwarf and T dwarf are highly preferable and can be used in a delightfully ambiguous way because they can refer to either a brown dwarf or a dwarf star.

How do we test the theoretical assertions about the nature of these objects? By definition, distinguishing a brown dwarf from a star requires knowledge that the object is not burning hydrogen in its core. There is no direct way to probe the interiors of these objects, but the most common indirect method used is the lithium test (Rebolo, Martín, \& Magazzù 1992). This test recognizes the fact

\footnotetext{
${ }^{4}$ Objects above $\sim 0.013 M_{\odot}$ are thought to burn heavy hydrogen (deuterium) for periods of $<100$ Myr early in their lives, but this does not halt further collapse for very long because the primordial abundance of deuterium is low (Burrows et al. 1997).
} 
that the temperature needed to sustain core hydrogen fusion in the lowest mass stars [ $T>3 \times 10^{6} \mathrm{~K}$ (Burrows et al. 1997; Nelson, Rappaport, \& Joss 1993)] is only slightly higher than that needed to burn lithium $\left(T \approx 2 \times 10^{6} \mathrm{~K}\right.$; Pozio 1991). If lithium does not burn, that means hydrogen is also not being fused. Once destroyed, lithium is not easily manufactured again in stellar interiors, so stars and brown dwarfs will never have more than their natal abundance of this element. The presence or absence of lithium in the spectrum can thus provide an indirect probe of inner temperatures.

As pointed out in Kirkpatrick et al. (1999), there are two important points to consider when applying the lithium test. First, it assumes that the objects are fully convective so that the photosphere (being measured by the spectra) and interior (which is what we hope to probe) are well mixed. This assumption may fail if the atmosphere breaks up into radiative zones. However, results from Stauffer, Schultz, \& Kirkpatrick (1998) show that there is a distinct "lithium edge" in the Pleiades; objects of type of M6.5 or earlier show no lithium, whereas those with cooler types do. The fact that lithium depletion shows such an abrupt edge in spectral type provides strong evidence that the coolest Pleiads are fully convective and that even at their young ages $(\sim 125 \mathrm{Myr})$ have had enough time to destroy completely their initial lithium content. Any cooler brown dwarf must also have passed through a late-M phase like these Pleiads are currently doing, so it was once fully convective and would have had sufficient time to destroy its lithium content if core temperatures were high enough. So even if cooler brown dwarfs begin to form radiative zones, the lithium test should still apply.

The second important point is that searching for the ground state $\mathrm{Li} \mathrm{I}$ line at $6708 \AA$ should prove futile below $T_{\text {eff }} \approx 1500 \mathrm{~K}$ because of the formation of $\mathrm{Li}$ bearing molecules like $\mathrm{LiCl}$ and $\mathrm{LiOH}$ (Lodders 1999). As shown in Figure 10, however, any object having temperatures this cold is a brown dwarf by default, so the lithium test need not be applied to judge its substellarity.

An unfortunate aspect of the lithium test, however, is that the $6708 \AA$ line is located in a portion of the spectrum where the flux is quite low. Large telescopes with moderate resolution optical spectrographs are required. Nonetheless, a set of optical spectra of 92 field L dwarfs was taken at the $10 \mathrm{~m}$ Keck Observatory and were analyzed by Kirkpatrick et al. (2000). It was found that the percentage of $\mathrm{L}$ dwarfs with discernible lithium absorption (equivalent widths $\geq 4 \AA$ ) is $\sim 0 \%$ at L0 then slowly rises to $20 \%$ by L2 and to $40 \%$ by L5. By L6-L8 roughly $50 \%-70 \%$ show lithium absorption. Measures of the lithium equivalent width appear to reach a maximum around L6 and then diminish at later types. At face value this could be attributed to atomic lithium disappearing into $\mathrm{LiCl}$; in fact, Lodders (1999) suggests that $\mathrm{Li}$ and $\mathrm{LiCl}$ should have equal abundances near $1500 \mathrm{~K}-1550 \mathrm{~K}$, which fits in well with the values of $T_{\text {eff }}$ measured for late-L dwarfs. However, this measure is a pseudo-equivalent width made against a relative continuum; at $6708 \AA$ this continuum is modulated by the strong ground state line of $\mathrm{Na}$ I to the blue and by the strong K I line to the red (see Figure 1). So, the decline in the measured lithium strength at late-L types may not indicate that the abundance of 
lithium is dropping but rather that the contrast of the line against a more heavily absorbed continuum is weakening.

The dotted orange line in the lower panel of Figure 10 shows the dividing line between lithium-burning objects and non-lithium-burning ones as predicted by theory (Chabrier et al. 2000). Objects to the right of this line have burned their primordial complement of lithium; those on the left have not. At ages greater than $\sim 400 \mathrm{Myr}$, the track coincides with the evolutionary track of a brown dwarf of mass $0.060 M_{\odot}$. Brown dwarfs of lower masses never burn their lithium regardless of age.

Qualitatively, the predictions agree with observations in the sense that an increasing percentage of $\mathrm{L}$ dwarfs at later type should be found to have lithium in their spectra. The tracks in Figure 10 suggest that dwarfs near L0 would represent a mixture of low-mass, lithium-depleted stars at ages greater than $250 \mathrm{Myr}$, high-mass lithium-depleted brown dwarfs at ages from 200-250 Myr, and lowermass lithium-rich brown dwarfs of various masses at ages less than $200 \mathrm{Myr}$. For a population of objects drawn from the field, we would expect very few to have ages below $200 \mathrm{Myr}$, so the finding that $\sim 0 \%$ of L0 dwarfs show lithium is not surprising. Figure 10 also suggests that the latest $\mathrm{L}$ dwarfs are comprised solely of brown dwarfs; those with ages exceeding 1-2 Gyr will have consumed their lithium, whereas younger ones will not have consumed their lithium. It should be noted that these older, lithium-depleted brown dwarfs lie in a very narrow range of masses, $0.060 M_{\odot}<M<0.072 M_{\odot}$, whereas the lithium-rich brown dwarfs represent all masses less than $0.060 M_{\odot}$. Hence, these lower-mass objects should dominate the numbers at late- $\mathrm{L}$, and the percentage of late-L dwarfs with lithium should be high. This, again, is in qualitative agreement with observations. Checking the quantitative agreement between observation and theory will not be possible until the field brown dwarf mass function at all masses has been measured.

A slightly less direct, though no less important, test of substellarity is to obtain dynamical mass measurements for $\mathrm{L}$ and $\mathrm{T}$ dwarfs. In order to judge the predictive ability of a plot such as Figure 10, though, it would be ideal to have systems in which the age has been measured through other means. This is possible for tight $\mathrm{L} / \mathrm{T}$ doubles that share common proper motion with a higher mass main sequence star. If the age and metallicity of the main sequence star have been measured or deduced via other means, then those same values can be assumed to apply to the $\mathrm{L} / \mathrm{T}$ doubles as well, leaving fewer unknowns and adding more leverage against which to gauge the robustness of the models. Such on-sky laboratories for this experiment exist $-\epsilon$ Ind $\mathrm{Ba} / \mathrm{Bb}$, Gl 564BC, and possibly $\mathrm{Gl} 417 \mathrm{~B}(\mathrm{C}$ ?)—but no results have been published so far.

When this review was written, only one object with a confirmed L or T spectral type has had its mass measured through dynamical means. Bouy et al. (2004) have measured the total mass of the L0.5 double 2MASSW J0746425 + 2000321AB to be $0.146_{-0.006}^{+0.016} M_{\odot}$. Bouy et al. (2004) measured only a relative orbit for the system, so the mass ratio cannot be measured without resorting to theory. Because the magnitude difference between the two components in non-zero-in fact, those 
authors derive spectral types of $\mathrm{L} 0$ and $\mathrm{L} 1.5$ for the two members - we can surmise that the A component has a mass somewhat greater than $0.073 M_{\odot}$ and the $\mathrm{B}$ component a mass somewhat less than $0.073 M_{\odot}$. The tracks in Figure 10 would therefore suggest that this system has an age of $\sim 250-500$ Myr. Given the present set of empirical data, an age this young cannot be ruled out although an older age would be considered more likely because the object is a field dwarf.

Lane et al. (2001; see also Kenworthy et al. 2001) present dynamical mass measurements for the late-M dwarf double $\mathrm{Gl} 569 \mathrm{Ba} / \mathrm{Bb}$, presumably only slightly warmer than the 2MASS J0746 +2000 system. They find a total mass of $0.123 \pm$ $0.02 M_{\odot}$ for this M8.5/M9 pair, but again measure only a relative orbit and thus lack the ability to measure a mass ratio. In this case, it is likely the Ba component is slightly more massive than $0.062 M_{\odot}$ and the $\mathrm{Bb}$ component slightly less massive than $0.062 M_{\odot}$. The tracks in Figure 10 would then suggest an age below $\sim 300 \mathrm{Myr}$, which again seems oddly too young for a field system. (The primary, Gl 569A, is an early-M dwarf with a poorly constrained age estimate itself.) Additional monitoring observations of other low-mass dwarfs in close binaries will provide other much needed checks of the theory and help determine if improbably young, theoretically determined ages are common in a larger sample.

4.3.2. NUMBER Using the relation of absolute magnitude versus spectral type (as in Figure 9), the discovery rate of $\mathrm{L}$ and $\mathrm{T}$ dwarfs from large surveys such as 2MASS and DENIS can be converted into space densities. The number of $\mathrm{L}$ dwarf discoveries from Kirkpatrick et al. (1999) gives an L dwarf space density of $(10 \pm 2) \times 10^{-3} / p c^{3}$ (Burgasser 2001), based on 17 objects; based on 7 other $\mathrm{L}$ dwarfs, Gizis et al. (2000) have calculated the space density of objects with optical types in the range L0-L4.5 as $(2.11 \pm 0.92) \times 10^{-3} / p c^{3}$; Cruz et al. (2003) find a similar value of $1.9 \times 10^{-3} / p c^{3}$ for L0-L4 based on a much larger sample. These values are uncorrected for selection effects such as Malmquist bias and unresolved binarity, deficiencies that Cruz et al. (in preparation) are remedying by using a more complete census of 2MASS-selected L dwarfs within $20 \mathrm{pc}$ of the Sun.

For T dwarfs, Burgasser (2001) derived a bias-corrected value of $\sim 21 \times 10^{-3} /$ $p c^{3}$ for the T5-T8 range using $14 \mathrm{~T}$ dwarfs identified from 2MASS. Placed in qualitative terms, a random slice of our Galaxy would show far more $\mathrm{T}$ dwarfs (even in this narrow spectral range) than $\mathrm{L}$ dwarfs. These numbers can be compared to the density of other types using the fairly complete census of $\mathrm{O}$ through $\mathrm{M}$ dwarfs (and also white dwarfs) within $8 \mathrm{pc}$ of the Sun and north of Dec $=-30^{\circ}$. The space density of these earlier type stars is $(93 \pm 8) \times 10^{-3} / p c^{3}$, and $70 \%$ of this number density is comprised of a single spectral type, the M dwarfs (see Kirkpatrick 2001b).

We can use these space densities of field $\mathrm{L}$ and $\mathrm{T}$ dwarfs to ask if brown dwarfs are a significant contributor to the mass budget of the Galaxy. Reid et al. (1999) made the first attempt at determining the mass function of field $\mathrm{L}$ and $\mathrm{T}$ dwarfs. Brown dwarfs, however, introduce an additional level of complexity not present when computing a mass function for field stars because they do not have a one-to-one mapping of spectral type into mass. An age is needed before assigning 
a mass to a brown dwarf, and age estimates are notoriously difficult to estimate for isolated field objects.

To circumvent this problem, Reid et al. (1999) assumed that the stellar/substellar birthrate is constant with time, that the mass function can be described by a power law of the form $\Psi(M) \propto M^{-\alpha}$, and that the Burrows et al. (1997) models are an accurate representation of brown dwarf evolution. With these assumptions in place, samples of stars and brown dwarfs were produced for each of the various assumed power law exponents. Then, the 2MASS selection criteria and magnitude limits were applied to the samples to produce a typical "observed" subset for each power law exponent. These observed subsets were compared to the actual observational sample of 2MASS late-M, L, and T dwarfs available in 1999. Reid et al. (1999) found that power laws with $1<\alpha<2$ provided the best matches to the empirical data.

Using a value of $\alpha=1.3$ (considered the most likely value by Reid 1999), we can extrapolate the mass function down to $0.010 M_{\odot}$, far below the detection limits of current surveys. Such an extrapolation is limited by the actual minimum mass of stellar/substellar formation, which could be far different from $0.010 M_{\odot}$, and the actual shape of the mass function, which the power law assumption may poorly match. Nonetheless, these assumptions suggest that roughly two-thirds of the objects within $8 \mathrm{pc}$ of the Sun are undetectable even with 2MASS. Despite their large numbers, though, these brown dwarfs are predicted to make up only about $10 \%$ of the mass that stars do (Reid 1999; Kirkpatrick 2001b).

More sophisticated Monte Carlo simulations have been produced by Burgasser (2004b) using five different assumed birthrates and six different forms of the mass function, five of which are power law distributions with $\alpha=0.0,0.5,1.0,1.5$, and 2.0 and the other a log-normal distribution. Despite the variety of forms assumed, Burgasser (2004b) finds some common traits amongst the resulting distributions of objects. First, $\mathrm{L}$ dwarfs are rare relative to $\mathrm{M}$ dwarfs and T dwarfs because mid-L is the location of the stellar/substellar transition at late ages; brown dwarfs with similar ages and with masses just below this transition have had sufficient time to cool to later types and thus are no longer L dwarfs. Second, the number densities of $\mathrm{T}$ (and cooler) dwarfs rise toward a low-luminosity peak that depends on the adopted minimum cutoff mass for the formation of brown dwarfs. The location of this peak may be measurable by the next generation of large-area surveys described in Section 6. The reader is referred to Chabrier (2003) and Allen et al. (2005) for other simulations.

\section{ADDITIONAL PARAMETERS IN SPECTRAL CLASSIFICATION}

In Section 2 the philosophy of spectral classification was discussed, leading to the establishment of schemes for $\mathrm{L}$ and $\mathrm{T}$ dwarfs in Section 3. The physical parameters giving rise to the $\mathrm{L}$ and $\mathrm{T}$ spectral sequences were then coaxed from the data 
and compared to theoretical models in Section 4. While most $\mathrm{L}$ and $\mathrm{T}$ dwarfs fit within the framework of the classification schemes addressed in earlier sections, a few do not. If the spectrum of such an odd object is thought of as a frame in a movie, to reuse the analogy from Section 3.2, then it becomes clear that this frame belongs to a different movie, not the one played by the standard $\mathrm{L}$ and $\mathrm{T}$ sequence. The quandary, then, is to explain the physical processes causing this odd object to appear different from the rest. Objects that defy ready classification do not highlight a failure of the classification system; rather, one of the strengths of a useful classification scheme is in easily identifying differences so they can be studied and explanations sought.

Several groups have noted spectra that appear unusual. In a couple of cases, the oddity was explained as a hybrid spectrum of a double system where one component is a mid- to late- $\mathrm{L}$ dwarf and the other a $\mathrm{T}$ dwarf. Because the absolute magnitudes (see $M_{J}$ in Figure 9 for example) of objects in this range can be very similar, the secondary may have an equal or greater contribution to the overall flux than the primary does depending upon the wavelength being considered. When the spectral shapes are as different as those of a late- $\mathrm{L}$ and a mid-T in the near-infrared, the hybrid spectrum can be very unusual. Such is thought to be the most likely explanation for 2MASS J05185995-2828372, an unusual object that has near-infrared $\mathrm{CH}_{4}$ and $\mathrm{CO}$ bandstrengths unlike any normal $\mathrm{L}$ or T dwarf (Cruz et al. 2004). The discrepant spectral types (as noted in Cruz et al. 2003) for SDSSp J042348.57-041403.5-L7.5 in the optical and T0 in the near-infrared-may also be explained by binarity (as predicted by Golimowski et al. 2004) because this object is double on Hubble Space Telescope images (A.J. Burgasser, private communication). McLean et al. (2003) find more subtle oddities in the spectra of DENIS-P J1228-1547 and DENIS-P J0205-1159; these oddities may again be due, at least in part, to binarity as both are known doubles.

In other cases, the explanation is not as clear. Kirkpatrick et al. (2000), using a data set of 92 optical spectra, identified two that defied normal classification. 2MASSW J1841086 + 311727 (L4 pec) shows an optical slope too blue for the type implied by its bandstrengths, and 2MASSW J2208136 + 292121 (L2 pec) shows anomalously narrow K I and anomalously strong TiO. McLean et al. (2003) note that Kelu-1 appears unusual in its near-infrared K I line strengths (other oddities noted by McGovern, priv. comm.), as do 2MASSI J1726000 + 153819 and 2MASSW J2244316 + 204343. The latter object is the reddest L dwarf known in $J-K_{s}$ color $(2.48 \pm 0.15)$ and also shows unusually strong $\mathrm{CO}$ bands near $2.3 \mu \mathrm{m}$ and a strangely shaped H-band continuum (McLean et al. 2003). Nakajima, Tsuji, \& Yanagisawa (2004) also note a strangely shaped H-band peak for the midL dwarf SDSSp J224953.45 + 004404.2 along with a weak $1.1 \mu \mathrm{m} \mathrm{H}_{2} \mathrm{O}$ band and weak near-infrared K I lines. These H-band continuum shapes in 2MASSW $\mathrm{J} 2244+2043$ and SDSS J2249 + 0044, which peak near $1.70 \mu \mathrm{m}$, are reminiscent of the odd triangular $\mathrm{H}$-band shapes seen in the spectra of Orion brown dwarf targets observed by Lucas et al. (2001). 
At least three T dwarfs also defy the trend suggested by other objects. 2MASS $\mathrm{J} 09373487+2931409$, which is the bluest known $\mathrm{T}$ dwarf in $J-K_{s}$ color $(-0.89 \pm 0.24)$, shows a very suppressed $2.1 \mu \mathrm{m}$ peak, an anomalously red slope between 8000 and 10,000 $⿱$ A, and unusually strong FeH absorption at $9896 \AA$ (Burgasser et al. 2002a, 2003b). SDSS J111010.01 + 011613.1 is noted for having strong K I lines for its spectral type (Knapp et al. 2004); the opposite effect is seen in the spectrum of 2MASS J00345157 + 0523050 (Burgasser et al. 2004a).

Perhaps the weirdest object of the unusual ones uncovered so far is 2MASS $\mathrm{J} 05325346+8246465$ (Burgasser et al. 2003a). This is a very peculiar L dwarf with colors like that of a mid-T dwarf $-J-K_{s}=0.26 \pm 0.16$. Unlike T dwarfs, where methane absorption drives the near-infrared colors blueward, the blue colors of this object appear to be caused by enormously strong collision-induced absorption by $\mathrm{H}_{2}$ throughout the $\mathrm{H}$ - and $\mathrm{K}$-bands. Both the optical and near-infrared spectra also show overly strong absorption by hydride molecules. What is causing this and the other objects noted above to stray from the normal trend?

In the previous two sections, we have dealt primarily with $\mathrm{L}$ and $\mathrm{T}$ dwarfs pulled from the field population, whose average age and metallicity are thought to be very similar to Solar values. However, varying either the age (which, as discussed below, equates to a change in gravity) or the metallicity of an L/T dwarf should have a noticeable effect on its spectral energy distribution. Some, if not all, of the oddities noted in the last two paragraphs may be explainable once these effects are considered. In the rest of this section, we will explore how the spectral classification of late-M, L, and T dwarfs can be further expanded to address these issues.

\subsection{First Parameter: Temperature (and Clouds)}

As discussed in Section 4.1 and Section 4.2, temperature is still the main driver of spectral shape in the $\mathrm{L}$ and $\mathrm{T}$ dwarf regimes, with the exception of the late- $\mathrm{L}$ to mid-T range where the behavior of clouds becomes a dominant effect. This first parameter, captured in the classification as spectral class + subtype (i.e., as L0, L4.5, T8, etc.), becomes the core against which we can extend the types. Figure 11 is a visualization of this expanded classification system in a three-axis parameter space. The temperature/clouds parameter is shown along the $\mathrm{x}$-axis. The other two axes, representing the gravity and metallicity parameters, are further discussed below.

\subsection{Second Parameter: Gravity}

Any slice along a fixed temperature in Figure 10 results in a range of objects of various masses and ages. In other words, at a given spectral subclass such as L0 $\left(T_{\text {eff }} \approx 2500 \mathrm{~K}\right)$ in our core classification, a varied assortment of objects can be found. If read off the top panel of Figure 10, we find that L0 dwarfs range from old, stably burning stars of fixed mass (mass $\approx 0.085 M_{\odot}$, age $>$ a few Gyr); to moderate-age, high-mass brown dwarfs (mass $\approx 0.075 M_{\odot}$, age $\approx$ a 


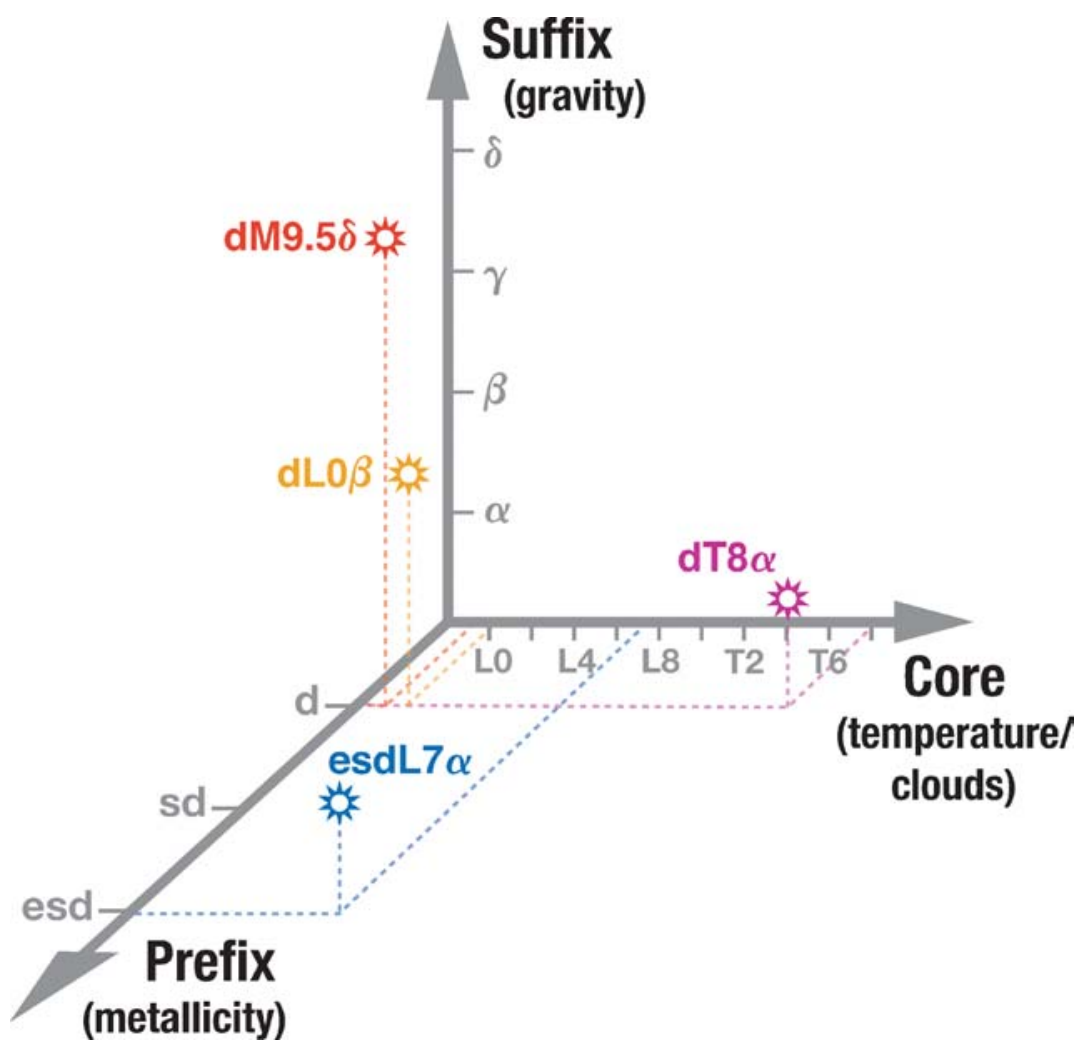

Figure 11 A schematic diagram illustrating a possible extension of late-M, L, and T dwarf classifications to three parameters. The three-part spectral types would include prefix + core + suffix, where each of the parts is determined via comparison to a suite of on-sky calibration standards. The core is thought to measure temperature and clouds, the prefix metallicity, and the suffix gravity. Example spectral types are shown in color on the Figure. The nomenclature given here is for demonstration purposes only but nonetheless highlights where spectral typing of the future may lead.

few $\times 100 \mathrm{Myr}$ ); to very young, low-mass brown dwarfs (mass $<0.020 \mathrm{M}_{\odot}$, age $<20 \mathrm{Myr}$ ). To first order, objects from low-mass stars through the lowest mass brown dwarfs are believed to have similar radii, so the gravities depend upon the mass. However, at a given spectral type the lowest mass brown dwarfs will not have contracted into their final configuration, meaning that these objects are larger than their higher mass counterparts at the same temperature. This enhances the difference in gravity between the high-mass and low-mass members in any spectral bin. As shown in figure 9 of Burrows et al. (1997), $\log (g)$ (in cm/s) in the late-L/early-T range can vary from 3.5 for a 3-Myr-old brown dwarf of mass $0.005 M_{\odot}$ to 5.5 for a $3-G y r-o l d$ brown dwarf of mass $0.075 M_{\odot}$. Such a large 
range in gravity should manifest itself through telltale signatures in the spectra. In essence, these signatures provide a way to chronometer brown dwarfs using spectroscopy alone.

Empirically we can build a grid of spectra to quantify gravity effects by looking at late-M, L, and $\mathrm{T}$ dwarfs across a wide spread of age-in star forming regions and clusters ranging from the Orion Nebula (age $\approx \mathrm{a}$ few Myr) to the Pleiades (age $\approx 125 \mathrm{Myr}$ ), as companions to young field stars (see, for example, the list in Kirkpatrick et al. 2001), and as old field objects (age $\approx$ a few Gyr). Presently, few very young $\mathrm{L}$ and $\mathrm{T}$ dwarfs are known ${ }^{5}$; late-M dwarfs, because they are brighter and thus easier to discover, have been the objects of choice in the empirical analysis done so far. Steele \& Jameson (1995) used the weakness of the Na I lines in their spectra of late-M Pleiades brown dwarf candidates to argue for companionship in the cluster; Martín, Rebolo, \& Zapatero Osorio (1996) further show that Pleiades brown dwarf candidates exhibit unusual VO strengths and pseudocontinuum slopes compared to field objects of the same spectral type; Luhman, Liebert, \& Rieke (1997) constructed hybrid spectra from a suite of observational standards to show that their late-M $\rho$ Oph brown dwarf candidate has gravity features intermediate between dwarfs and giants. Many other papers using gravity-sensitive features as indicators of youth have appeared in the literature since these earlier works.

Although the importance of gravity markers in the spectra of cluster brown dwarfs has a rich history as noted above, Gorlova et al. (2003) made the first large-scale attempt at quantifying these effects through near-infrared observations of giants $(\log (g) \approx 0)$, late-M-type brown dwarfs in young clusters, and brown dwarfs in the field. Those authors found that the $\mathrm{H}_{2} \mathrm{O}$ band at $1.35 \mu \mathrm{m}$ and $\mathrm{CO}$ band at $2.30 \mu \mathrm{m}$ were relatively insensitive to gravity for $\mathrm{M}$ dwarfs and could be used as good measures of temperature (i.e., our core spectral type). Lines of K I at $1.25 \mu \mathrm{m}$ and $\mathrm{Na} \mathrm{I}$ at $2.21 \mu \mathrm{m}$ along with possibly the $\mathrm{FeH}$ band at $1.20 \mu \mathrm{m}$ are very gravity sensitive. Fortunately, these gravity markers are easily recognizable at the coarse resolutions commonly employed for classification and thus can be incorporated directly into the spectral type once such schemes are developed. Figure 11 shows gravity as the second parameter (y-axis) in the three-pronged classification of late-M, L, and T dwarfs.

\subsection{Third Parameter: Metallicity}

The evolutionary tracks displayed in Figure 10 are for solar metallicity. Low-mass stars and brown dwarfs produced during the earliest epochs of star formation in the Galaxy will have had much lower abundances of metals. Burrows et al. (1993)

\footnotetext{
${ }^{5}$ Doubts persist regarding the youth of the $\mathrm{L}$ dwarfs and one $\mathrm{T}$ dwarf purported to lie in the $\sigma$ Ori cluster (Zapatero Osorio et al. 1999, 2000, 2002; Martín et al. 2001; Barrado y Navascués 2002; Martín \& Zapatero Osorio 2003). McGovern et al. (2004) argue that the L1.5 dwarf SOri47 shows none of the signs of lower gravity as originally reported by Zapatero Osorio et al. (1999), and Burgasser et al. (2004b) argue that the T dwarf SOri70 is more likely a field object based on more careful analysis of the original data.
} 
show that the transition from star to brown dwarf in a zero-metallicity environment is around $0.094-0.098 M_{\odot}$ and $T_{\text {eff }} \approx 3500 \mathrm{~K}$, considerably higher than for solar metallicity objects. Saumon et al. (1994) find that the major opacity sources in low-temperature, zero-metallicity dwarfs are collision-induced absorption by $\mathrm{H}_{2}$, Rayleigh scattering by $\mathrm{H}_{2}$ and $\mathrm{H}$, and bound-free and free-free absorption by $\mathrm{H}^{-}$. Rayleigh scattering is the dominant opacity source throughout the visible, and the dominance of collision-induced absorption by $\mathrm{H}_{2}$ in the near-infrared gives rise to very blue $J-K_{s}$ colors.

In reality, low-metallicity $\mathrm{L}$ and $\mathrm{T}$ dwarfs will fall somewhere between these two extremes. Although theory is a useful guide, actual on-sky anchor stars are needed before a classification scheme can be produced. Because these low-metallicity objects will have been formed at very early ages, they will be members of the thick disk or halo and hence will in general have higher space motions than their younger, higher metallicity counterparts. Perhaps the most efficient way of finding these objects is performing a proper motion survey over a very large area of the sky. The first such surveys probing into this regime are using existing photographic plate material at R- and I-bands. The survey by Lépine, Shara, \& Rich (2002) has uncovered one low-metallicity dwarf tentatively classified as early-L (Lépine, Rich, \& Shara 2003), and Scholz et al. (2004) have found one low-metallicity dwarf as late as M9.5.

Ideally, these surveys should be conducted at near-infrared wavelengths where these objects are brightest. Such a proper motion survey could be conducted using coverage provided by 2MASS and DENIS at J- or K-bands, although unfortunately the two epochs would be nearly contemporaneous and could only identify objects of the highest motions. Nonetheless, the near-infrared is already proving a fertile ground for the discovery of such objects. In their spectroscopic followup of photometrically selected T-dwarf candidates, Burgasser et al. (2003a) have found not only the aforementioned 2MASS J05325346 + 8246465-believed to be a low-metallicity brown dwarf member of the halo from its spectral signature and kinematics-but also two other low-metallicity L dwarfs, 2MASS $\mathrm{J} 16262034+3925190$ (Burgasser 2004a) and possibly 2MASS J00412179 + 3547133 (Burgasser et al. 2004a).

Therefore, a third parameter is needed in the classification of these cool objects. Figure 11 shows metallicity as the third component (z-axis) of the three-parameter spectral classification.

\subsection{Applications}

Developing a three-parameter classification is in its infancy, but the classifications could look something like this: The core class could be augmented with a suffix indicating the gravity type and a prefix indicating the metallicity type. In both cases the indices will be divided into natural groupings as warranted by the empirical data. The suite of spectra being collected by Kirkpatrick (optical) and McGovern (near-infrared) suggests that perhaps four gravity classes can be distinguished at 
low to moderate resolution. For the purposes of this review, we will tentatively call these gravity suffixes $\alpha, \beta, \gamma$, and $\delta$, where $\alpha$ refers to the highest gravity and $\delta$ the lowest. The metallicity index already has a precedent from the study of M subdwarfs, where Gizis (1997) advocates the use of prefixes $d$, sd, and esd, where $d$ represents solar metallicity, $s d$ moderately low metallicity, and $e s d$ very low metallicity.

As demonstrated in Figure 11, for example, an old, high-gravity, solar-metallicity T dwarf like Gl 570D might be designated as “ $d T 8 \alpha$." The early-L dwarf Roque 25 (Martín et al. 1998) in the Pleiades has the signature of slightly lower gravity than early-L dwarfs in the field and might be designated as " $d L 0 \beta$." The even lower gravity Taurus brown dwarf KPNO-Tau 4 (Briceño et al. 2002) might be designated “ $d M 9.5 \delta$." An object like the very low-metallicity L dwarf 2MASS J0532 + 8246 might receive the temporary designation " $(e) s d L 7: \alpha$ " which would be revised once enough other $\mathrm{L}$ subdwarfs are found to make a convincing classification sequence. Clearly, not all of the three-dimensional parameter space shown in Figure 11 would be occupied with objects because, for instance, low-metallicity (population II) objects will always have higher gravities than high-metallicity (population I) objects of the same temperature.

In conclusion, the classification of $\mathrm{L}$ and $\mathrm{T}$ dwarfs can be expanded to become more all-encompassing. The spectra contain a great deal of information not currently encapsulated in the core spectral type alone, and it is hoped that an extension of the classification to include other parameters will make $\mathrm{L}$ and $\mathrm{T}$ spectral types even more useful to researchers in the future.

\section{PROSPECTS FOR THE FUTURE}

Brown dwarfs of lower temperature than the coolest known $\mathrm{T}$ dwarf undoubtedly exist. Spectroscopic models by Burrows, Sudarsky, \& Lunine (2003) suggest that at temperatures somewhat below the current floor of $\sim 750 \mathrm{~K}$ (see Table 3 ) several physical processes occur that alter the emergent spectra. The strong optical lines of $\mathrm{Na} I$ and $\mathrm{K} I$ disappear around $500 \mathrm{~K}$, water clouds form around $400 \mathrm{~K}-500 \mathrm{~K}$, the $J-K$ color reverses (to the red) between $300 \mathrm{~K}$ and $400 \mathrm{~K}$, the position of the Mband peak shifts at these same low temperatures, and ammonia clouds form below $\sim 160 \mathrm{~K}$. Counterintuitively, though, the formation of water and ammonia clouds has little effect on the spectral energy distribution, and it might be one of the other effects that provides a natural spectroscopic division between spectral classes. If, after studying the empirical data, a new spectral type beyond $\mathrm{T}$ is warranted, Kirkpatrick et al. (1999) have suggested "Y" as the new letter designation. It is worth noting that there is no reason for the $\mathrm{T}$ class to end at T9.5, either. The data may show that more $\mathrm{T}$ dwarf subdivisions are needed before another natural break point is reached, and having the T sequence extend to T11 or T15 or later before the next new type ("Y0") is used is perfectly legitimate. Nature will let us know.

How will such cooler objects be found? If history serves as a guide, the first of the "Y" dwarfs is likely to be found as a companion to a known, nearby star or brown 
dwarf. Now that many observatories are deploying adaptive optics systems that improve our resolution capabilities from the ground, the hunt for close companions around nearby stars can proceed in a way not easily possible before. Space-based capabilities such as the near- and mid-infrared imaging made possible by the Spitzer Space Telescope enable searches at wavelengths tuned to the peak flux of these objects. However, history has also shown that studying brown dwarfs en masse takes dedicated large-area surveys; companion searches are likely to produce only a few such examples. As further described below, several large-area surveys planned for the next decade aim to go deeper than present-day 2MASS, DENIS, and SDSS.

The United Kingdom Infrared Deep Sky Survey (UKIDSS; http://www.ukidss. org) will map $\sim 7200$ square degrees of northern sky from the United Kingdom Infrared Telescope atop Mauna Kea, Hawaii. The survey, which will be done primarily at JHK bands and probe down to $5 \sigma$ depths of $K \geq 18.4$, is a combination of several surveys, three of which are large area (>1000 square degrees). All three include plans to redo their entire surveyed area in one of the bands at a second epoch to measure proper motions. The UKIDSS has an anticipated start date of 2005 and will take seven years to complete.

The Visible and Infrared Survey Telescope for Astronomy (VISTA; http://www. vista.ac.uk) will be a $4 \mathrm{~m}$ telescope located at the Cerro Paranal Observatory site in Chile. It is viewed as a modern replacement of the $1.2 \mathrm{~m}$ United Kingdom Schmidt telescope whose goal was to provide photographic imaging of the entire southern sky. The goal of VISTA is to provide near-infrared imaging and then (if funds permit) optical imaging to deep depths using infrared arrays and CCDs. Because this will be a digital catalog of the entire southern sky at near-infrared and perhaps also optical wavelengths, it will be of use to brown dwarf researchers both as a search tool and a comparison data set for other surveys. The near-infrared sky will be mapped in two bands reaching $5 \sigma$ depths of $J=20.2$ and $K_{s}=18.4$, and second epoch to the same depth is planned at a later time (Emerson, priv. comm.). Deeper surveys covering smaller areas of sky are also envisioned. Delivery of the telescope and infrared camera is slated for 2006 and the first surveys are expected to take 12 years to complete.

The Panoramic Survey Telescope and Rapid Response System (Pan-STARRS; http://pan-starrs.ifa.hawaii.edu) is being constructed primarily to find near-Earth asteroids but will be very useful for brown dwarf science as well. It will consist of four $1.8-\mathrm{m}$ telescopes, each of which image the same piece of sky simultaneously. The multi-telescope design is favored over a single telescope of the same collecting area due to design and cost considerations. The cameras, equipped with CCDs and fitted with a suite of optical filters, will reach a depth of $\sim 24$ th magnitude. The site will likely be in Hawaii either at Haleakala on Maui or at Mauna Kea on the Big Island. The telescopes will survey $\sim 6000$ square degrees per clear night, meaning that the entire observable sky can be mapped roughly three times each month. One telescope will be built as a prototype and is expected to have first light on Haleakala in 2006. The many multiple epochs of this survey can be used both as a proper motion finding tool and as a means for 
acquiring direct trigonometric parallaxes of all nearby stars and brown dwarfs.

The Large Synoptic Survey Telescope (LSST; http://www.lsst.org) is a groundbased 8.4-m telescope that will map the entire observable sky $(\sim 20,000$ square degrees, at a site yet to be selected) every three nights. The main science drivers are cataloging Solar System objects and identifying high-z QSOs. The camera will take images in five filters $(g, r, i, z$, and $Y)$ simultaneously to single-pass $10 \sigma$ equivalent limiting magnitudes of $\mathrm{V}=24$ and $\mathrm{I}=23$. The $i, z$, and $Y$ filters cover the entire range from 7000 to $10400 \AA$, so the survey probes sufficiently deeply at long enough wavelengths that it should be very valuable for studies of L, T, and cooler dwarfs. Like Pan-STARRS, data across the time domain can be used to identify faint proper motion objects and to measure trigonometric parallaxes directly. First light is expected in 2012.

As ambitious as each of these surveys is, none covers the entire sky and all are done from the ground, limiting us to observations within the terrestrial "windows" dictated by Earth's atmosphere. The Wide-field Infrared Survey Explorer (WISE; http://wise.ssl.berkeley.edu) remedies both of these problems. Finding the nearest, coolest brown dwarfs is one of WISE's primary goals, and the mission is designed specifically with brown dwarfs in mind. It is a dedicated space-based observatory with a 40-cm telescope and a four-channel camera that will operate at 3.5, 4.6, 12, and $23 \mu \mathrm{m}$. These first two bands are ideally suited to finding very cool brown dwarfs because the $3.5 \mu \mathrm{m}$ band sits squarely in the methane fundamental band and the $4.6 \mu \mathrm{m}$ band sits atop the area of highest flux in the substellar spectrum (Burrows, Sudarsky \& Lunine 2003). The resulting red 3.5-4.7 $\mu \mathrm{m}$ colors are expected to be uniquely characteristic of cool brown dwarfs, enabling them to be selected against the background of other objects. The expected launch date is 2009.

These surveys underscore the fact that research on L dwarfs, T dwarfs, and their even cooler counterparts has a very promising future.

\section{CONCLUSIONS}

Less than a decade ago, only two objects were known that would eventually carry designations of either L dwarf or T dwarf. Today almost 500 of these are recognized, opening a new branch of astronomy on objects with spectra different than anything previously known.

At the genesis of any field of study, deciphering order amongst the chaos is the first step in interpreting new findings. For L and T dwarfs this ordering has been provided through the empirical observation that their spectra can be arranged in a sequential fashion, linking them seamlessly to the spectral sequence seen for stars from type $\mathrm{O}$ through type $\mathrm{M}$. With this sequence in hand, spectral typing schemes in the spirit of the MK Process have been established for L and T dwarfs. These types provide both a vernacular for easy reference and a framework against which to begin to understand the governing physics. 
Observations of large numbers of $\mathrm{L}$ and $\mathrm{T}$ dwarfs have revealed several important facts:

- The spectra of these objects are replete with atomic and molecular absorptions. Most dominant among these are lines of neutral alkali metals and bands of metal oxides, metal hydrides, water, methane, and ammonia.

- Known L dwarfs span the effective temperature range from $\sim 2500 \mathrm{~K}$ to $\sim 1300 \mathrm{~K}$; $\mathrm{T}$ dwarfs can be as warm as $\sim 1500 \mathrm{~K}$, with the coolest known example having $T_{\text {eff }} \approx 750 \mathrm{~K}$.

- As with $\mathrm{O}$ through $\mathrm{M}$ stars, temperature is the major factor determining the spectral type.

- The optical spectral ordering provides a better map into effective temperature than the near-infrared spectral ordering, but both sequences show that temperature changes very little from early- to mid-T types.

- T dwarfs far outnumber L dwarfs in the Solar Neighborhood, but there are more $\mathrm{O}$ - through $\mathrm{M}$-type stars in a given volume than there are $\mathrm{L}$ through $\mathrm{T}$ dwarfs.

Theoretical interpretations also suggest the following:

- For L dwarfs, the spectral shapes cannot be matched unless dust grains, produced by the condensation of atomic and molecular species, are suspended in the photosphere.

- Mid- to late-T dwarfs cannot be properly reproduced unless this dust is assumed to have vanished from the photosphere.

- Several rival theories have been put forth to explain the process of cloud clearing between these two extremes (the late-L to mid-T transition). Observations have shown that the temperatures in this transition area are nearly constant (with large scatter), so the parameter mainly responsible for determining the spectral type may be cloud fraction, percentage of condensate rainout, gravity, or a combination of each of these.

- L dwarfs are believed to be a combination of very low-mass, hydrogen burning stars and brown dwarfs, whereas all $\mathrm{T}$ dwarfs are believed to be substellar. Empirical observations via the lithium test support these assertions but cannot directly state whether an object is burning hydrogen stably or not.

- Masses for L dwarfs with solar metallicity are predicted to be as large as $\sim 0.085 M_{\odot}$. The scant observational evidence available at present (dynamical mass measures for one late-M dwarf pair and one early-L dwarf pair) suggests this value may be $\sim 10 \%$ too high. T dwarfs may have masses as large as $\sim 0.075 M_{\odot}$, but no empirical mass measurements have yet been made to test this.

- Extrapolations of the mass function of $\mathrm{L}$ and $\mathrm{T}$ dwarfs suggest that there may be roughly twice as many brown dwarfs as stars in the Solar 
Neighborhood, but this number depends critically on the low-mass cutoff for brown dwarf formation.

The study of L dwarfs, T dwarfs, and their even cooler counterparts has an exciting future. Spectra of a few oddball $\mathrm{L}$ and $\mathrm{T}$ dwarfs highlight the fact that our current one-dimensional spectral types are still somewhat inadequate. An extension to a second and third dimension may provide a more universal scheme for categorizing (and understanding) these objects. Several future ground-based and space-based missions will provide fertile hunting grounds for brown dwarfs cooler than type $\mathrm{T}$ as well, assuring us that if Nature produces a continuum of spectra between late-T dwarfs and brown dwarfs of temperatures akin to Jupiter, then the gap may soon be filled.

\section{ACKNOWLEDGMENTS}

The author would like to thank George Helou (IPAC) for providing partial salary support during the writing of this manuscript. The author is also indebted to Peter Allen, Adam Burgasser, Kelle Cruz, Michael Cushing, Jim Emerson, Chris Gelino, Sandy Leggett, Jim Liebert, Michael Liu, Mark McGovern, Ian McLean, Tadashi Nakajima, Keith Noll, Ben Oppenheimer, Neill Reid, Tom Roellig, and Didier Saumon for valuable discussions, use of data sets, and/or access to papers prior to their publication. The construction of Table 1, Table 3, and Figures 8-9 relied heavily on NASA's Astrophysics Data System (ADS) Bibliographic Services and the NASA/IPAC Infrared Science Archive (IRSA). The latter is operated by the Jet Propulsion Laboratory, California Institute of Technology, under contract with the National Aeronautics and Space Administration. Almost all of the spectra illustrated in Figures 1-5 were obtained atop Mauna Kea on the Big Island of Hawaii. The author wishes to recognize and acknowledge the very significant cultural role and reverence that the summit of Mauna Kea has always had within the indigenous Hawaiian community. He and his colleagues consider themselves most fortunate to have had the opportunity to conduct observations from this mountain.

\section{The Annual Review of Astronomy and Astrophysics is online at http://astro.annualreviews.org}

\section{LITERATURE CITED}

Ackerman AS, Marley MS. 2001. Ap. J. 556: $872-84$

Allard F, Hauschildt PH, Alexander DR, Tamanai A, Schweitzer A. 2001. Ap. J. 556:357-72

Allard NF, Allard F, Hauschildt PH, Kielkopf
JF, Machin L. 2003. Astron. Astrophys. 411: L473-76

Allen PR, Koerner DW, Reid IN, Trilling DE. 2005. Ap. J. In press

Allende Prieto C, Lambert DL, Asplund M. 2001. Ap. J. 556:L63-66 
Anders E, Grevesse N. 1989. Geochim. Cosmochim. Acta 53:197-214

Baraffe I, Chabrier G, Barman TS, Allard F, Hauschildt PH. 2003. Astron. Astrophys. 402:701-12

Barrado y Navascués D, Zapatero Osorio MR, Martín EL, Béjar VJS, Rebolo R, Mundt R. 2002. Astron. Astrophys. 393:L85-88

Basri G, Mohanty S, Allard F, Hauschildt PH, Delfosse X, et al. 2000. Ap. J. 538:363-85

Becklin EE, Zuckerman B. 1988. Nature 336: 656-58

Berriman B, Kirkpatrick D, Hanisch R, Szalay A, Williams R. 2003. In Large Telescopes and Virtual Observatory: Visions for the Future, 25th Meet. IAU, Jt. Discuss. 8, 17 July, Sydney, Aust.

Biemont E, Baudoux M, Kurucz RL, Ansbacher W, Pinnington EH. 1991. Astron. Astrophys. 249:539-44

Boccaletti A, Chauvin G, Lagrange A-M, Marchis R. 2003. Astron. Astrophys. 410: 283-8

Borysow A, Jorgensen UG, Zheng C. 1997. Astron. Astrophys. 324:185-95

Bouy H, Brandner W, Martín EL, Delfosse X, Allard F, Basri G. 2003. Astron. J. 126:152654

Bouy H, Duchene G, Kohler R, Brandner W, Bouvier J, et al. 2004. Astron. Astrophys. 423:341-52

Briceño C, Luhman KL, Hartmann L, Stauffer JR, Kirkpatrick JD. 2002. Ap. J. 580:317-35

Burgasser AJ. 2001. The discovery and characterization of methane-bearing brown dwarfs and the definition of the T spectral class. $\mathrm{PhD}$ thesis. Calif. Inst. Technol.

Burgasser AJ. 2004a. Ap. J. 614:L73-76

Burgasser AJ. 2004b. Astron. J. Suppl. Ser. 155:191-207

Burgasser AJ, Kirkpatrick JD, Brown ME, Reid IN, Burrows A, et al. 2002a. Ap. J. 564:42151

Burgasser AJ, Kirkpatrick JD, Brown ME, Reid IN, Gizis JE, et al. 1999. Ap. J. 522:L65-68

Burgasser AJ, Kirkpatrick JD, Burrows A, Liebert J, Reid IN, et al. 2003a. Ap. J. 592:1186-92
Burgasser AJ, Kirkpatrick JD, Cutri RM, McCallon H, Kopan G, et al. 2000a. Ap. J. 531:L57-60

Burgasser AJ, Kirkpatrick JD, Liebert J, Burrows A. 2003b. Ap. J. 594:510-24

Burgasser AJ, Kirkpatrick JD, McElwain MW, Cutri RM, Burgasser AJ, Skrutskie MF. 2003c. Astron. J. 125:850-57

Burgasser AJ, Kirkpatrick JD, McGovern MR, McLean IS, Prato L, Reid IN. 2004a. Ap. J. 604:827-31

Burgasser AJ, Marley MS, Ackerman AS, Saumon D, Lodders K, et al. 2002b. Ap. J. 571:L15-54

Burgasser AJ, McElwain MW, Kirkpatrick JD. 2003d. Astron. J. 126:2487-94

Burgasser AJ, McElwain MW, Kirkpatrick JD, Cruz KL, Tinney CG, Reid IN. 2004b. Astron. J. 127:2856-70

Burgasser AJ, Wilson JC, Kirkpatrick JD, Skrutskie MF, Colonno MR, et al. 2000b. Astron. J. 120:1100-5

Burrows A, Hubbard WB, Lunine JI, Liebert J. 2001. Rev. Mod. Phys. 73:719-65

Burrows A, Hubbard WB, Saumon D, Lunine JI. 1993. Ap. J. 406:158-71

Burrows A, Marley M, Hubbard WB, Lunine JI, Guillot T, et al. 1997. Ap. J. 491:85675

Burrows A, Ram RS, Bernath P, Sharp CM, Milsom JA. 2002. Ap. J. 577:986-92

Burrows A, Sharp CM. 1999. Ap. J. 512:843-63

Burrows A, Sudarsky D, Lunine JI. 2003. Ap. J. 596:587-96

Burrows A, Volobuyev M. 2003. Ap. J. 583: 985-95

Carson JC, Eikenberry SS, Brandl BB, Wilson JC, Hayward TL. 2002. Bull. Am. Astron. Soc. $34: 1176$

Chabrier G. 2003. Publ. Astron. Soc. Pac. 115: 763-95

Chabrier G, Baraffe I. 1997. Astron. Astrophys. 327:1039-53

Chabrier G, Baraffe I, Allard F, Hauschildt P. 2000. Ap. J. 542:464-72

Chauvin G, Lagrange A-M, Dumas C, Zuckerman B, Mouillet D, et al. 2004. Astron. Astrophys. 425:L29-32 
Close LM, Siegler N, Freed M, Biller B. 2003. Ap. J. 587:407-22

Cruz KL, Burgasser AJ, Reid IN, Liebert J. 2004. Ap. J. 604:L61-64

Cruz KL, Reid IN, Liebert J, Kirkpatrick JD, Lowrance PJ. 2003. Astron. J. 126:2421-48

Cuby JG, Saracco P, Moorwood AFM, D’Odorico S, Lidman C, et al. 1999. Astron. Astrophys. 349:L41-44

Cushing MC, Rayner JT, Davis SP, Vacca WD. 2003. Ap. J. 582:1066-72

Cushing MC, Rayner JT, Vacca WD. 2005. Ap. $J$. In Press

Dahn CC, Harris HC, Vrba FJ, Guetter HH, Canzian B, et al. 2002. Astron. J. 124:117089

Delfosse X, Tinney CG, Forveille T, Epchtein N, Bertin E, et al. 1997. Astron. Astrophys. 327:L25-28

Delfosse X, Tinney CG, Forveille T, Epchtein N, Borsenberger J, et al. 1999. Astron. Astrophys. Suppl. 135:41-56

Dulick M, Bauschlicher CW, Burrows A, Sharp CM, Ram RS, Bernath P. 2003. Ap. J. 594: 651-63

Els SG, Sterzik MF, Marchis F, Pantin E, Endl M, Kürster M. 2001. Astron. Astrophys. 370:L1-4

Epchtein N, Deul E, Derriere S, Bosenberger J, Egret D, et al. 1999. Astron. Astrophys. 349:236-42

Fan X, Knapp GR, Strauss MA, Gunn JE, Kupton RH, et al. 2000. Astron. J. 119:928-35

Freed M, Close LM, Siegler N. 2003. Ap. J. 584:453-58

Garrison RF. 1995. Publ. Astron. Soc. Pac. 107:507-12

Geballe TR, Knapp GR, Leggett SK, Fan X, Golimowski DA, et al. 2002. Ap. J. 564:46681

Gizis JE. 1997. Astron. J. 113:806-22

Gizis JE. 2002. Ap. J. 575:484-92

Gizis JE, Kirkpatrick JD, Wilson JC. 2001. Astron. J. 121:2185-88

Gizis JE, Monet DG, Reid IN, Kirkpatrick JD, Liebert J, Williams RJ. 2000. Astron. J. 120:1085-99

Gizis JE, Reid IN, Knapp GR, Liebert J, Kirk- patrick JD, et al. 2003. Astron. J. 125:330210

Goldman B, Delfosse X, Forveille T, Afonso C, Alard C, et al. 1999. Astron. Astrophys. 351: L5-9

Golimowski DA, Leggett SK, Marley MS, Fan X, Geballe TR, et al. 2004. Astron. J. 127:3516-36

Gorlova NI, Meyer MR, Rieke GH, Liebert J. 2003. Ap. J. 593:1074-92

Goto M, Kobayashi N, Terada H, Gaessler W, Kazawa T, et al. 2002. Ap. J. 567:L59-62

Grevesse N, Lambert DL, Sauval AJ, van Dishoeck EF, Farmer CB, Norton RH. 1990. Astron. Astrophys. 232:225-30

Grevesse N, Lambert DL, Sauval AJ, van Dishoek EF, Farmer CB, Norton RH. 1991. Astron. Astrophys. 242:488-95

Hall PB. 2002. Ap. J. 580:L77-78

Hawley SL, Covey KR, Knapp GR, Golimowski DA, Fan XH, et al. 2002. Astron. J. 123:3409-27

Hayashi C, Nakano T. 1963. Prog. Theor. Phys. 30:460-74

Holweger H, Bard A, Kock M, Kock A. 1991. Astron. Astrophys. 249:545-49

Kendall TR, Delfosse X, Martín EL, Forveille T. 2004. Astron. Astrophys. 416:L17-20

Kendall TR, Mauron N, Azzopardi M, Gigoyan K. 2003. Astron. Astrophys. 403:929-36

Kenworthy M, Hofmann KH, Close L, Hinz P, Mamajek E, et al. 2001. Ap. J. 554:L6770

Kirkpatrick JD. 1998. In Brown Dwarfs and Extrasolar Planets, ed. R Rebolo, ASP Conf. Ser. 134:405-15. San Francisco: Astron. Soc. Pac.

Kirkpatrick JD. 2001a. In Ultracool Dwarfs: New Spectral Types L and T, ed. HRA Jones, IA Steele, pp. 139-52. New York: Springer

Kirkpatrick JD. 2001b. In Tetons 4: Galactic Structure, Stars and the Interstellar Medium, ed. CE Woodward, MD Bicay, JM Shull, ASP Conf. Ser. 231:17-35

Kirkpatrick JD, Beichman CA, Skrutskie MF. 1997. Ap. J. 476:311-18

Kirkpatrick JD, Dahn CC, Monet DG, Reid IN, Gizis JE, et al. 2001. Astron. J. 121:3235-53 
Kirkpatrick JD, Henry TJ, Liebert J. 1993. Ap. J. 406:701-7

Kirkpatrick JD, Reid IN, Liebert J, Cutri RM, Nelson B, et al. 1999. Ap. J. 519:802-33

Kirkpatrick JD, Reid IN, Liebert J, Gizis JE, Burgasser AJ, et al. 2000. Astron. J. 120:44772

Knapp GR, Leggett SK, Fan X, Marley MS, Geballe TR, et al. 2004. Astron. J. 127:355378

Kumar SS. 1963a. Ap. J. 137:1121-25

Kumar SS. 1963b. Ap. J. 137:1126-28

Lane BF, Zapatero Osorio MR, Britton MC, Martín EL, Kulkarni SR. 2001. Ap. J. 560: 390-99

Leggett SK, Allard F, Geballe TR, Hauschildt PH, Schweitzer A. 2001. Ap. J. 548:908-18

Leggett SK, Geballe TR, Fan X, Schneider DP, Gunn JE, et al. 2000. Ap. J. 536:L35-38

Leggett SK, Golimowski DA, Fan XH, Geballe TR, Knapp GR, et al. 2002. Ap. J. 564:45265

Lépine S, Rich RM, Shara MM. 2003. Ap. J. 591:L49-52

Lépine S, Shara MM, Rich RM. 2002. Astron. J. 124:1190-212

Liebert J, Kirkpatrick JD, Cruz KL, Reid IN, Burgasser A, et al. 2003. Astron. J. 125:34347

Liu MC, Fischer DA, Graham JR, Lloyd JP, Marcy GW, Butler RP. 2002a. Ap. J. 571: 519-27

Liu MC, Wainscoat R, Martín EL, Barris B, Tonry J. 2002b. Ap. J. 568:L107-11

Lodders K. 1999. Ap. J. 519:793-801

Lodders K. 2002. Ap. J. 577:974-85

Lodders K, Fegley B. 2002. Icarus 155:393424

Lodieu N, Caux E, Monin J-L, Klotz A. 2002. Astron. Astrophys. 383:L15-18

Lodieu N, Scholz R-D, McCaughrean MJ. 2002. Astron. Astrophys. 389:L20-23

Lucas PW, Roche PF, Allard F, Hauschildt PH. 2001. MNRAS 326:695-721

Luhman KL, Liebert J, Rieke GH. 1997. Ap. J. 489:L165-68

Marley MS, Seager S, Saumon D, Lodders K, Ackerman AS, et al. 2002. Ap. J. 568:335-42
Martín EL, ed. 2003. Brown Dwarfs, IAU Symp. Ser., 211

Martín EL, Basri G, Delfosse X, Forveille T. 1997. Astron. Astrophys. 327:L29-32

Martín EL, Basri G, Zapatero Osorio MR, Rebolo R, López RJG. 1998. Ap. J. 507:L4144

Martín EL, Delfosse X, Basri G, Goldman B, Forveille T, et al. 1999. Astron. J. 118:246682

Martín EL, Rebolo R, Zapatero Osorio MR. 1996. Ap. J. 469:706-14

Martín EL, Zapatero Osorio MR. 2003. Ap. J. 593:L113-16

Martín EL, Zapatero Osorio MR, Barrado y Navascués D, Béjar VJS, Rebolo R. 2001. Ap. J. 558:L117-21

Matthews K, Nakajima T, Kulkarni SR, Oppenheimer BR. 1996. Astron. J. 112:1678-882

McGovern MR, Kirkpatrick JD, McLean IS, Burgasser AJ, Prato L, Lowrance PJ. 2004. Ap. J. 600:1020-24

McLean IS, McGovern MR, Burgasser AJ, Kirkpatrick JD, Prato L, Kim SS. 2003. Ap. J. 596:561-86

McLean IS, Prato L, Kim SS, Wilcox MK, Kirkpatrick JD, Burgasser A. 2001. Ap. J. 561:L115-18

Metchev SA, Hillenbrand LA. 2004. Ap. J. 617:1330-46

Mihalas D. 1984. In The MK Process and Stellar Classification, ed. RF Garrison, pp. 4-16. Toronto: David Dunlap Obs.

Morgan WW, Keenan PC. 1973. Annu. Rev. Astron. Astrophys. 11:29-50

Morgan WW, Keenan PC, Kellman E. 1943. An Atlas of Stellar Spectra with an Outline of Spectral Classification. Chicago, IL: Univ. Chicago Press

Nakajima T, Oppenheimer BR, Kulkarni SR, Golimowski DA, Matthews K, et al. 1995. Nature 378:463-65

Nakajima T, Tsuji T, Yanagisawa K. 2004. Ap. J. 607:499-510

Nelson LA, Rappaport S, Joss PC. 1993. Ap. J. 404:723-33

Noll KS, Geballe TR, Leggett SK, Marley MS. 2000. Ap. J. 541:L75-78 
Noll KS, Geballe TR, Marley MS. 1997. Ap. J. 489:L87-90

Oppenheimer BR, Kulkarni SR, Matthews K, Nakajima T. 1995. Science 270:1478-79

Oppenheimer BR, Kulkarni SR, Matthews K, van Kerkwijk MH. 1998. Ap. J. 502:93243

Potter D, Martín EL, Cushing MC, Baudoz P, Brandner W, et al. 2002. Ap. J. 567:L13336

Pozio F. 1991. Mem. Soc. Astron. Ital., 62:17179

Rebolo R, Martín EL, Magazzù A. 1992. Ap. J. 389:L83-86

Rebolo R, Zapatero Osorio MR, Madruga S, Béjar VJS, Arribas S, Licandro J. 1998. Science 282:1309-12

Reid IN. 1999. In Star Formation 1999, ed. T Nakamoto, Nobeyama Radio Obs., pp. 327 32

Reid IN, Burgasser AJ, Cruz KL, Kirkpatrick JD, Gizis JE. 2001. Astron. J. 121:171021

Reid IN, Kirkpatrick JD, Gizis JE, Dahn CC, Monet DG, et al. 2000. Astron. J. 119:36977

Reid IN, Kirkpatrick JD, Leibert J, Burrows A, Gizis JE, et al. 1999. Ap. J. 521:613-29

Roellig TL, Van Cleve JE, Sloan GC, Wilson JC, Saumon D, et al. 2004. Ap. J. Suppl. 154:418-21

Ruiz MT, Leggett SK, Allard F. 1997. Ap. J. 491:107-10

Salim S, Lépine S, Rich RM, Shara MM. 2003. Ap. J. 586:L149-52

Saumon D, Bergeron P, Lunine JI, Hubbard WB, Burrows A. 1994. Ap. J. 424:333-44

Saumon D, Geballe TR, Leggett SK, Marley MS, Freedman RS, et al. 2000. Ap. J. 541:374-89

Saumon D, Marley MS, Lodders K, Freedman RS. 2003. See Martín 2003, pp. 345-53

Schneider DP, Knapp GR, Hawley SL, Covey KR, Fan XH, et al. 2002. Astron. J. 123:45865

Scholz R-D, Lehmann I, Matute I, Zinnecker H. 2004. Astron. Astrophys. 425:519-27

Scholz R-D, McCaughrean MJ, Lodieu N,
Kuhlbrodt B. 2003. Astron. Astrophys. 398: L29-33

Scholz R-D, Meusinger H. 2002. MNRAS 336: L49-52

Stanway ER, Bunker AJ, McMahon RG, Ellis RS, Treu T, McCarthy PJ. 2004. Ap. J. 607:704-20

Stauffer JR, Schultz G, Kirkpatrick JD. 1998. Ap. J. 499:L199-202

Steele IA, Jameson RF. 1995. MNRAS 272:630-46

Stephens DC. 2003. See Martín 2003, pp. 35558

Strauss MA, Fan X, Gunn JE, Leggett SK, Geballe TR, et al. 1999. Ap. J. 522:L61-64

Testi L, D'Antona F, Ghinassi F, Licandro J, Magazzu A, et al. 2001. Ap. J. 552:L14750

Thorstensen JR, Kirkpatrick JD. 2003. Publ. Astron. Soc. Pac. 115:1207-10

Tinney CG, Burgasser AJ, Kirkpatrick JD. 2003. Astron. J. 126:975-92

Tsuji T. 2000. In Very Low-Mass Stars and Brown Dwarfs, ed. R Rebolo, MR ZapateroOsorio, pp. 156-68. Cambridge, UK: Cambridge Univ. Press

Tsuji T. 2002. Ap. J. 575:264-90

Tsuji T, Nakajima T. 2003. Ap. J. 585:L15154

Tsuji T, Nakajima T, Yanagisawa K. 2004. Ap. J. 607:511-29

Tsvetanov ZI, Golimowski DA, Zheng W, Geballe TR, Leggett SK, et al. 2000. Ap. J. 531:L61-65

van Altena WF, Lee JT, Hoffleit D. 1995. Yale Trigonometric Parallaxes. New Haven, CT: Yale Univ. Observatory. 4th ed.

Volk K, Blum R, Walker G, Puxley P. 2003. IAU Circ. 8188:2

Vrba FJ, Henden AA, Luginbuhl CB, Guetter HH, Munn JA, et al. 2004. Astron. J. 127:2948-68

Wilson JC, Kirkpatrick JD, Gizis JE, Skrutskie MF, Monet DG, Houck JR. 2001. Astron. J. 122:1989-2000

Wilson JC, Miller NA, Gizis JE, Skrutskie MF, Houck JR, et al. 2003. See Martín 2003, pp. 197-200 
York DG, Adelman J, Anderson JE Jr, Anderson SF, Annis J, et al.1999. Astron. J. 120:157987

Zapatero Osorio MR, Béjar VJS, Martín EL, Rebolo R, Barrado y Navascués D, et al. 2000. Science 290:103-7

Zapatero Osorio MR, Béjar VJS, Martín EL,
Rebolo R, Barrado y Navascués D, et al. 2002. Ap. J. 578:536-42

Zapatero Osorio, MR, Béjar VJS, Rebolo R, Martín EL, Basri G. 1999. Ap. J. 524:L11518

Zuckerman B, Becklin EE. 1992. Ap. J. 386: 260-64 